\title{
Deeply-bound $K^{-} p p$ state in the ${ }^{3} \mathrm{He}\left(\right.$ in-flight $\left.K^{-}, n\right)$ spectrum and its moving pole near the $\pi \Sigma N$ threshold
}

\author{
Takahisa Koike*1 and Toru Harada ${ }^{\dagger 2}$ \\ ${ }^{1}$ Advanced Meson Science Laboratory, \\ RIKEN Nishina Center, Wako-shi, Saitama 351-0198, Japan \\ ${ }^{2}$ Research Center for Physics and Mathematics, \\ Osaka Electro-Communication University, \\ Neyagawa, Osaka, 572-8530, Japan
}

(Dated: May 26, 2018)

\begin{abstract}
The formation of a deeply-bound $K^{-} p p$ state with $I=1 / 2, J^{\pi}=0^{-}$by the ${ }^{3} \mathrm{He}(\mathrm{in}-\mathrm{flight}$ $\left.K^{-}, n\right)$ reaction is theoretically investigated in a distorted-wave impulse approximation using the Green's function method. The expected inclusive and semi-exclusive spectra at $p_{K^{-}}=1.0$ $\mathrm{GeV} / \mathrm{c}$ and $\theta_{\mathrm{lab}}=0^{\circ}$ are calculated for the forthcoming J-PARC E15 experiment. We demonstrate these spectra with several phenomenological $K^{-}$- " $p p$ " optical potentials $U^{\text {opt }}(E)$ which have an energy-dependent imaginary part multiplied by a phase space suppression factor, fitting to recent theoretical predictions or experimental candidates of the $K^{-} p p$ bound state. The results show that a cusp-like peak at the $\pi \Sigma N$ threshold is an unique signal for the $K^{-} p p$ bound state in the spectrum including the $\left[K^{-} p p\right] \rightarrow Y+N$ decay process from the two-nucleon $K^{-}$absorption, as well as a distinct peak of the $K^{-} p p$ bound state. The shape of the spectrum is explained by a trajectory of a moving pole of the $K^{-} p p$ bound state in the complex energy plane. The importance of the $\left[K^{-} p p\right] \rightarrow Y+N$ spectrum is emphasized in order to extract clear evidence of the $K^{-} p p$ bound state.
\end{abstract}

PACS numbers: $25.80 . \mathrm{Nv}, 13.75 . \mathrm{Jz}, 36.10 . \mathrm{Gv}, 21.45 .-\mathrm{v}$

\footnotetext{
* E-mail:tkoike@riken.jp

† E-mail:harada@isc.osakac.ac.jp
} 


\section{INTRODUCTION}

The antikaon-nucleon $(\bar{K} N)$ interaction in nuclei is very important to elucidate the nature of high dense nuclear matter [1]. Since the $\bar{K} N I=0$ interaction is believed to be strongly attractive, one would expect the existence of deeply-bound $\bar{K}$ nuclear states [2]. Especially, a three-body $\bar{K} N N$ (unstable) bound state with a $\left[\bar{K} \otimes\{N N\}_{I=1}\right]_{I=1 / 2}, J^{\pi}=0^{-}$ configuration, which is called " $K^{-} p p$ " symbolically, is suggested to be the lightest and the most fundamental $\bar{K}$ nucleus.

In 1963, Nogami [3] firstly discussed a possible existence of the $K^{-} p p$ bound state by a rather crude calculation. About 40 years later, Yamazaki and Akaishi [4] restarted to study the structure of the $K^{-} p p$ bound state based on a quantitative few-body calculation with a phenomenological $\bar{K} N$ interaction which reproduces the mass and width of $\Lambda(1405)$ as a $\bar{K}-N$ quasibound state. They predicted that the binding energy and width for the $K^{-} p p$ state are B.E. $=48 \mathrm{MeV}$ and $\Gamma=61 \mathrm{MeV}$, respectively. Many other theoretical works [512] have also supported the existence of the $K^{-} p p$ bound state, but the predicted binding energies and widths are not converged (see Fig. 2); Shevchenko, Gal and Mareš [5] performed a $\bar{K} N N-\pi \Sigma N$ coupled-channel Faddeev calculation using phenomenological $\bar{K} N-\pi \Sigma$ interactions, leading to B.E. $=55-70 \mathrm{MeV}$ and $\Gamma=95-110 \mathrm{MeV}$. Ikeda and Sato [6] also obtained B.E. $=79 \mathrm{MeV}$ and $\Gamma=74 \mathrm{MeV}$ in a similar Faddeev calculation with a Chiral $\mathrm{SU}(3)$ based $\bar{K} N-\pi Y$ interaction. On the other hand, some authors claimed B.E. $\simeq 20$ $\mathrm{MeV}[7,11]$ with $\bar{K} N$ interactions based on the Chiral unitary approach $[13,14]$, which are less attractive than the phenomenological $\bar{K} N$ ones in the bound state region. The discrepancy between theoretical results perhaps come from an ambiguity of the $\bar{K} N$ interaction, together with a different procedure for a three-body calculation involving decay processes. Further theoretical investigations are apparently needed.

Recently, several experimental observations of the $K^{-} p p$ state have been reported: The data by the FINUDA collaboration at DA $\Phi$ NE [15] suggested evidence of a deeply-bound $K^{-} p p$ state in invariant-mass spectroscopy from stopped $K^{-}$reactions on ${ }^{6} \mathrm{Li},{ }^{7} \mathrm{Li}$ and ${ }^{12} \mathrm{C}$. Their measured energy and width are B.E. $=115 \pm 9 \mathrm{MeV}$ and $\Gamma=67_{-14}^{+16} \mathrm{MeV}$, respectively, whereas Magas et al. [16] claimed that the FINUDA data can be explained without postulating the existence of the $K^{-} p p$ bound state. The OBELIX experiment at LEARCERN [17] also suggested the observation of the $K^{-} p p$ state in invariant-mass spectroscopy 
from stopped $\bar{p}$ reactions on ${ }^{4}$ He. Very recently, Yamazaki et al. [18] have found new experimental events of the $K^{-} p p$ state in $p+p \rightarrow K^{+}+\Lambda+p$ reactions by a reanalysis of old DISTO experimental data at SATURNE-Saclay. However, these experimental results would also leave room for other interpretations and therefore more experimental data are required in order to confirm whether the $K^{-} p p$ system has a deeply-bound state or not.

Iwasaki et al. [19] have proposed a new experiment searching the deeply-bound $K^{-} p p$ state at J-PARC by the missing-mass spectrum of the ${ }^{3} \mathrm{He}\left(\right.$ in-flight $\left.K^{-}, n\right)$ reaction, together with invariant-mass spectra detecting all particles via decay processes from the $K^{-} p p$ bound state (J-PARC E15 experiment). Moreover, a measurement of the $K^{-} p p$ state in $p+p$ collisions has been planned by the FOPI collaboration at GSI [20], as proposed by Yamazaki and Akaishi [21]. A search for the light $\bar{K}$ nuclear systems involving the $K^{-} p p$ state in the stopped $K^{-}$reactions on ${ }^{3} \mathrm{He} /{ }^{4} \mathrm{He}$ targets has been also planned by the AMADEUS collaboration at DA $\Phi$ NE [22].

Our purpose is to theoretically clarify the expected inclusive and semi-exclusive spectra by the ${ }^{3} \mathrm{He}$ (in-flight $\left.K^{-}, n\right)$ reaction for the forthcoming J-PARC E15 experiment. In a previous work [23], we examined these spectra of the ${ }^{3} \mathrm{He}\left(\right.$ in-flight $\left.K^{-}, n\right)$ reactions within a distorted-wave impulse approximation (DWIA) employing the Green's function method [24] which well describes unstable hadron systems [25]. It has been shown that the ${ }^{3} \mathrm{He}$ (in-flight $\left.K^{-}, n\right)$ reaction provides a promising spectrum which contains a $s$-wave dominance in the $K^{-}$bound region, where a strong nuclear distortion for $K^{-}$is reduced [23]. This is a major advantage of a use of the $s$-shell nuclear target such as ${ }^{3} \mathrm{He}$. A similar calculation for the ${ }^{3} \mathrm{He}$ target is also presented by Yamagata et al. [11] with the help of the Chiral unitary approach. The investigations for heavier targets within a similar framework have been found in several publications [26-29]. In the case of $p$-shell targets of ${ }^{12} \mathrm{C}$ and ${ }^{16} \mathrm{O}$ [26], the signals of the $\bar{K}$ nuclear states would not be extracted due to their broad widths even if the bound states exist $[27,28]$.

In this paper, we theoretically investigate the formation and decay of the deeply-bound $K^{-} p p$ state by the ${ }^{3} \mathrm{He}\left(\right.$ in-flight $\left.K^{-}, n\right)$ reaction at the incident $K^{-}$momentum $p_{K^{-}}=$ $1.0 \mathrm{GeV} / \mathrm{c}$ and the forward direction $\theta_{\mathrm{lab}}=0^{\circ}$ within a DWIA. To search a signal of the deeply-bound $K^{-} p p$ state, we examine the inclusive and semi-exclusive spectra including 
one-nucleon $K^{-}$absorption processes,

$$
\left[K^{-} p p\right] \rightarrow " K^{-} p "+p \rightarrow \pi+Y+N
$$

and two-nucleon $K^{-}$absorption processes,

$$
\left[K^{-} p p\right] \rightarrow K^{-}+" p p " \rightarrow Y+N
$$

near the $\pi \Sigma N$ decay threshold, where $Y=\{\Sigma, \Lambda\}$. Since there exist many predictions of B.E. and $\Gamma$ for the $K^{-} p p$ state at present, we demonstrate typical spectra by using the $K^{-}{ }_{-} " p p "$ optical potential which reproduces the values of each B.E. and $\Gamma$ phenomenologically. Here we employ the phenomenological $K^{-}$- " $p p$ " optical potentials having an energydependence due to the phase space factors of the above processes (1) and (2). If B.E. is larger than about $100 \mathrm{MeV}$, a decay channel via the process (1) with $Y=\Sigma$ is kinematically closed, so that the decay width of the $K^{-} p p$ bound state would be considerably small. Indeed, recent Faddeev calculations [5, 6] or several experimental observations [15, 18] have suggested that B.E. is close to the energy at the $\pi \Sigma N$ decay threshold. In order to deal with such a threshold effect, we must take into account the energy dependence in the $K^{-}$- " $p p$ " optical potential. This is a natural extension of the previous work [23] where we mainly discussed the spectra with an energy-independent optical potential. A preliminary result of this subject is partially found in Ref. [30].

The outline of this paper is as follows: In Sect. II, we mention our DWIA framework using the Green's function method for the ${ }^{3} \mathrm{He}\left(\right.$ in-flight $\left.K^{-}, n\right)$ reaction, and we introduce several phenomenological energy-dependent $K^{--_{-}} " p p$ " optical potentials, of which parameters are determined to reproduce the values of B.E. and $\Gamma$ obtained from a recent few-body calculation $[4,5,7]$ or experimental candidate $[15,17,18]$. In Sect. III, we show the calculated inclusive and semi-exclusive spectra with each optical potential. We find a distinct peak structure or a cusp-like structure at the $\pi \Sigma N$ threshold in the spectrum depending on the potential parameters; its shape behavior of the spectrum is governed by a pole trajectory for the $K^{-} p p$ state in the complex energy plane. In Sect. IV, we discuss the dependence of the spectral shape on the potential parameters and the branching ratio of $K^{-}$absorptions systematically, in order to understand the appearance of a cusp-like spectrum. The summary and conclusion are given in Sect. V. 


\section{FRAMEWORK}

\section{A. Distorted-wave impulse approximation (DWIA)}

In the DWIA framework [31], the inclusive double-differential cross section of the ${ }^{3} \mathrm{He}(\mathrm{in}-$ flight $\left.K^{-}, n\right)$ reaction at the forward direction $\theta_{\text {lab }}=0^{\circ}$ in the lab system is written [32] as

$$
\frac{d^{2} \sigma}{d E_{n} d \Omega_{n}}=\beta\left(0^{\circ}\right)\left\langle\frac{d \sigma}{d \Omega_{n}}\left(0^{\circ}\right)\right\rangle_{\text {lab }}^{K^{-} N \rightarrow N \bar{K}} S(E),
$$

where $S(E)$ is a strength function for the $K^{-} p p$ system as a function of the energy $E$, and $\left\langle d \sigma / d \Omega_{n}\left(0^{\circ}\right)\right\rangle_{\text {lab }}^{K^{-}} N \rightarrow N \bar{K}$ is a Fermi-averaged cross section for the elementary $K^{-}+N \rightarrow N+\bar{K}$ forward scattering which is equivalent to a backward $\bar{K}+N$ elastic scattering in the lab system [33]. The lab cross section for the non-spin-flip $K^{-}+n \rightarrow n+K^{-}\left(K^{-}+p \rightarrow n+\bar{K}^{0}\right)$ process amounts to $24.5 \mathrm{mb} / \mathrm{sr}(13.1 \mathrm{mb} / \mathrm{sr})$ in free space [33, 34], and is reduced to 13.9 $\mathrm{mb} / \mathrm{sr}(7.5 \mathrm{mb} / \mathrm{sr})$ with Fermi-averaging [28, 35]. Both of the $K^{-}+n \rightarrow n+K^{-}$elastic scattering and the $K^{-}+p \rightarrow n+\bar{K}^{0}$ charge exchange reaction can contribute to the formation of the $K^{-} p p I=1 / 2$ state through the coupling between $K^{-} p p$ and $\bar{K}^{0} p n$ channels. Thus an incoherent sum of contributions from these $K^{-}+n \rightarrow n+K^{-}$and $K^{-}+p \rightarrow n+\bar{K}^{0}$ processes, as is done in Ref. [11], may be unsuitable for the $K^{-} p p$ bound region. In the $\bar{K}^{0} / K^{-}$charge basis, the coupled-channel calculation would be needed.

Here we consider the cross section of Eq. (3) in the isospin basis because total isospin $I=1 / 2$ is expected to be an almost good quantum number in the $K^{-} p p$ bound state. The contribution from the elementary processes is approximately estimated by the isoscalar

$\Delta I=0$ transition amplitude $f_{\Delta I=0}=-\sqrt{2 / 3}\left(f_{K^{-} n \rightarrow n K^{-}}+\frac{1}{2} f_{K^{-} p \rightarrow n \bar{K}^{0}}\right)$ including a spectroscopic factor for the $K^{-} p p I=1 / 2$ state formed on ${ }^{3} \mathrm{He}$ [28]. If we use the amplitude $f_{\Delta I=0}$ with Fermi-averaging at $p_{K^{-}}=1.0 \mathrm{GeV} / \mathrm{c}$ and $\theta_{\mathrm{lab}}=0^{\circ}$, the Fermi-averaged cross section $\left\langle d \sigma / d \Omega_{N}\left(0^{\circ}\right)\right\rangle_{\text {lab }}^{\Delta I=0}=\left|\left\langle f_{\Delta I=0}\right\rangle\right|^{2}$ is found to be $16.4 \mathrm{mb} / \mathrm{sr}$, whereas $1.2 \mathrm{mb} / \mathrm{sr}$ for the isovector $\Delta I=1$ transition [28]. In our calculations, therefore, we adopt $16.4 \mathrm{mb} / \mathrm{sr}$ as a value of $\left\langle d \sigma / d \Omega_{n}\left(0^{\circ}\right)\right\rangle_{\text {lab }}^{K^{-} N \rightarrow N \bar{K}}$ in Eq. (3).

The kinematical factor $\beta\left(0^{\circ}\right)[28,32]$ in Eq.(3) expresses the translation from the twobody $K^{-}$-n lab system to the $K^{-}{ }^{3} \mathrm{He}$ lab system at $\theta_{\text {lab }}=0^{\circ}[36]$, and it is defined as

$$
\beta\left(0^{\circ}\right)=\left(1-\frac{E_{n}^{(0)}}{E_{K^{-}}^{(0)}} \frac{p_{K^{-}}-p_{n}^{(0)}}{p_{n}^{(0)}}\right) \frac{p_{n} E_{n}}{p_{n}^{(0)} E_{n}^{(0)}},
$$


where $p_{K^{-}}$and $p_{n}\left(E_{K^{-}}\right.$and $\left.E_{n}\right)$ are momenta of the incident $K^{-}$and the emitting $n$ (energies of the residual $K^{-}$and the emitting $n$ in the final state) in the many-body $K^{-}$ $+{ }^{3} \mathrm{He} \rightarrow n+\left[K^{-} p p\right]$ reaction, respectively, and the quantities with an (0) superscript are in the two-body $K^{-}+n \rightarrow n+K^{-}$reaction. Note that the momentum transfer of this reaction becomes negative; $q\left(0^{\circ}\right) \equiv p_{K^{-}}-p_{n}<0$. For the negative momentum transfer, $\beta\left(0^{\circ}\right)$ enhances the spectrum of Eq.(3) by a factor $1-2$ depending on $p_{n}$ and $E_{K^{-}}[28]$.

The strength function of the $K^{-} p p$ system, $S(E)$, in Eq. (3) can be given as a function of the energy $E$ measured from the $K^{-}+p+p$ threshold;

$$
E=M_{K^{-} p p}-\left(M_{K^{-}}+M_{p}+M_{p}\right)
$$

where $M_{K^{-} p p}, M_{K^{-}}$and $M_{p}$ are the masses of the $K^{-} p p$ bound state, the $K^{-}$and the proton, respectively. In this calculations, we assumed a " $p p$ " pair as a rigid core with a ${ }^{1} S_{0}$ state. This assumption would be suitable for qualitatively describing the structure of the $K^{-} p p$ state, as long as we consider the deeply-bound region. A simple $(1 \mathrm{~s})^{3}$ harmonic oscillator model is used for the ${ }^{3} \mathrm{He}$ wave function, in which the relative $2 N-N$ wave function has the form of $\phi_{2 N-N}(r) \propto \exp \left(-r^{2} / 2 a^{2}\right)$ where $a=b_{N} \sqrt{3 / 2}$. The size parameter $b_{N}$ is taken to

be $1.30 \mathrm{fm}$, which reproduces the experimental r.m.s charge radius of ${ }^{3} \mathrm{He}, \sqrt{\left\langle r^{2}\right\rangle}=1.94$ $\mathrm{fm}[37]$.

\section{B. Green's function method}

Here we consider Green's function for the $K^{-} p p$ system. It is obtained by solving the Klein-Gordon equation numerically;

$$
\left\{\left(E-V_{\mathrm{Coul}}(\boldsymbol{r})\right)^{2}+\nabla^{2}-\mu^{2}-2 \mu U^{\mathrm{opt}}(E ; \boldsymbol{r})\right\} G\left(E ; \boldsymbol{r}, \boldsymbol{r}^{\prime}\right)=\delta^{3}\left(\boldsymbol{r}-\boldsymbol{r}^{\prime}\right),
$$

where $\mu$ is the reduced mass between the $K^{-}$and the " $p p$ " core-nucleus, and $V_{\text {Coul }}$ is the Coulomb potential with the finite nuclear size effect. $U^{\text {opt }}(E)$ is an energy-dependent $K^{-}$" $p p$ " optical potential between the $K^{-}$and the " $p p$ " core-nucleus, which is assumed to be the Lorentz scalar type.

According to the Green's function method [24], we can write $S(E)$ as

$$
S(E)=-\frac{1}{\pi} \operatorname{Im}\left[\sum_{\alpha, \alpha^{\prime}} \int d \boldsymbol{r} d \boldsymbol{r}^{\prime} f_{\alpha}^{\dagger}(\boldsymbol{r}) G_{\alpha, \alpha^{\prime}}\left(E ; \boldsymbol{r}, \boldsymbol{r}^{\prime}\right) f_{\alpha^{\prime}}\left(\boldsymbol{r}^{\prime}\right)\right]
$$


with

$$
f_{\alpha}(\boldsymbol{r})=\chi^{(-) *}\left(\boldsymbol{p}_{n}, \frac{M_{\mathrm{C}}}{M_{K^{-} p p}} \boldsymbol{r}\right) \chi^{(+)}\left(\boldsymbol{p}_{K^{-}}, \frac{M_{\mathrm{C}}}{M_{\mathrm{A}}} \boldsymbol{r}\right)\left\langle\alpha\left|\psi_{n}(\boldsymbol{r})\right| i\right\rangle,
$$

where $G_{\alpha, \alpha^{\prime}}(E)$ is the complete Green's function for the $K^{-} p p$ system, and $\left\langle\alpha\left|\psi_{n}(\boldsymbol{r})\right| i\right\rangle$ is the $2 N-N$ wave function for a struck neutron in the target where $\alpha$ denotes the complete set of eigenstates for the system. $\chi^{(+)}$and $\chi^{(-)}$are distorted waves of the incoming $K^{-}$with the momentum $\boldsymbol{p}_{K^{-}}$and the outgoing $n$ with $\boldsymbol{p}_{n}$, respectively. The factors of $M_{\mathrm{C}} / M_{K^{-}} p$ and $M_{\mathrm{C}} / M_{\mathrm{A}}$ in Eq.(8) take into account the recoil effects, where $M_{\mathrm{C}}$ and $M_{\mathrm{A}}$ are the masses of the " $p p$ " core-nucleus and the ${ }^{3} \mathrm{He}$ target, respectively. The recoil effects have to moderate a whole shape of the spectrum. Indeed, if the recoil factors are omitted, the cross section of a peak in the bound region is reduced by about 50\%; a yield in the quasi-free (QF) region grows up, and a QF peak is shifted upward to the higher-energy side and its width is broader. Here we actually use the factor of $M_{\mathrm{C}} / M_{K^{-} p p}$ in not only $\chi^{(-)}$but also $\chi^{(+)}$for simplicity. If we use an alternative factor of $M_{\mathrm{C}} / \bar{M}_{A K}$ where $\bar{M}_{A K}$ is the mean mass of $M_{\mathrm{A}}$ and $M_{K^{-}}$pp instead of $M_{\mathrm{C}} / M_{K^{-} p p}$, we find that the cross section of the peak in the bound region is enhanced by less than $10 \%$.

By Green's function technique, the strength function $S(E)$ for the inclusive spectrum can be easily decomposed into two parts [23, 24];

$$
S(E)=S^{\operatorname{con}}(E)+S^{\operatorname{esc}}(E)
$$

where $S^{\text {con }}(E)$ denotes the $K^{-}$conversion processes including the decay modes of $\left[K^{-} p p\right] \rightarrow$ $\pi+Y+N$ and $\left[K^{-} p p\right] \rightarrow Y+N$, which come from the one- and two-nucleon $K^{-}$absorptions in Eqs. (1) and (2), respectively; $S^{\text {esc }}(\mathrm{E})$ denotes the $K^{-}$escape processes where the $K^{-}$ leaves from the core-nucleus as $\left[K^{-} p p\right] \rightarrow K^{-}+$"pp" above the $K^{-}+p+p$ threshold $(E>0)$. By an abbreviated notation for $G(E), U^{\text {opt }}(E)$ and $f$ instead of those in Eq. (7), we have

$$
\begin{aligned}
S^{\mathrm{con}}(E) & =-\frac{1}{\pi}\left\langle f^{\dagger} G^{\dagger}(E)\left\{\operatorname{Im} U^{\mathrm{opt}}(E)\right\} G(E) f\right\rangle, \\
S^{\mathrm{esc}}(E) & =-\frac{1}{\pi}\left\langle f^{\dagger}\left(1+G^{\dagger}(E) U^{\mathrm{opt} \dagger}(E)\right)\left\{\operatorname{Im} G_{0}(E)\right\}\left(1+U^{\mathrm{opt}}(E) G(E)\right) f\right\rangle,
\end{aligned}
$$

where $G_{0}(E)$ is a free Green's function.

With the help of the eikonal approximation, we express the distorted waves in Eq. (8), 
as

$$
\begin{aligned}
\chi^{(-) *}\left(\boldsymbol{p}_{n}, \boldsymbol{r}\right) & =\exp \left[-i \boldsymbol{p}_{n} \cdot \boldsymbol{r}-\frac{i}{v_{n}} \int_{z}^{+\infty} U_{n}\left(\boldsymbol{b}, z^{\prime}\right) d z^{\prime}\right] \\
\chi^{(+)}\left(\boldsymbol{p}_{K^{-}}, \boldsymbol{r}\right) & =\exp \left[+i \boldsymbol{p}_{K^{-}} \cdot \boldsymbol{r}-\frac{i}{v_{K^{-}}} \int_{-\infty}^{z} U_{K^{-}}\left(\boldsymbol{b}, z^{\prime}\right) d z^{\prime}\right]
\end{aligned}
$$

with an impact parameter coordinate $\boldsymbol{b}$ and the optical potential for $\lambda=K^{-}$or $n$,

$$
U_{\lambda}(r)=-i \frac{v_{\lambda}}{2} \bar{\sigma}_{\lambda N}^{\mathrm{tot}}\left(1-i \alpha_{\lambda N}\right) \rho(r)
$$

where $\rho(r)$ is a nuclear density distribution, and $\bar{\sigma}_{\lambda N}^{\text {tot }}$ and $\alpha_{\lambda N}$ denote the isospin-averaged total cross section and the ratio of the real to imaginary parts of the forward amplitude for the $\lambda+N$ scattering, respectively. At $p_{K^{-}}=1.0 \mathrm{GeV} / \mathrm{c}$, the total cross sections of $\sigma_{K^{-} p}^{\text {tot }}$ and $\sigma_{K^{-} n}^{\text {tot }}$ amount to $\sim 50 \mathrm{mb}$ and $\sim 40 \mathrm{mb}$, respectively, and $\sigma_{n p}^{\text {tot }}$ varies within $30-40 \mathrm{mb}$ in the corresponding momenta $p_{n}=1.1-1.4 \mathrm{MeV} / \mathrm{c}$ [38]. We confirm that the absolute values of the formation cross section of Eq. (3) are enhanced or reduced by up to about $20 \%$ when the values of $\bar{\sigma}_{\lambda N}^{\text {tot }}$ are changed within $30-50 \mathrm{mb}$, but a whole shape of the spectrum is hardly

moderated. Thus we use $\bar{\sigma}_{K}^{\text {tot }}{ }^{-}{ }=\bar{\sigma}_{n N}^{\text {tot }}=40 \mathrm{mb}[33,39]$ for simplicity. Since the formation cross section is rather insensitive to the values of $\alpha_{\lambda N}$ [40], we use $\alpha_{K^{-N}}=\alpha_{n N}=0[33,39]$. This fact implies that the distortion effects are not so important in our calculation, because of the small nuclear size of the ${ }^{3} \mathrm{He}$ target. It has been shown that a distortion factor for the (in-flight $K^{-}, n$ ) reactions on ${ }^{3} \mathrm{He}$ is estimated as $D_{\text {dis }}=0.47$ for $1 s_{p} \rightarrow 1 s_{K^{-}}$transition [23], of which value is about 5 times as large as $D_{\text {dis }}=0.095$ for $1 p_{p} \rightarrow 1 s_{K^{-}}$transition on a ${ }^{12} \mathrm{C}$ target [39]. This is also an advantage of a use of the $s$-shell nuclear targets such as ${ }^{3} \mathrm{He}$.

\section{Optical potentials for the $K^{-}-" p p$ " system}

FIG. 1

In a previous paper [23], we evaluated the spectra with the energy-independent $K^{-}-^{-} " p p$ " optical potential which reproduces the result of the binding energy (B.E.) and width $(\Gamma)$ by Yamazaki and Akaishi [4] or by Shevchenko, Gal and Mareš [5]. On the other hand, Mares̆ et al. [41] introduced phase space suppression factors, $f_{1}^{Y}(E)$ and $f_{2}^{Y}(E)$, which denote for 
the one- and two-nucleon $K^{-}$absorption processes, respectively:

$$
\begin{aligned}
f_{1}^{Y}(E)= & \frac{M_{1}^{3}(0)}{M_{1}^{3}(E)} \sqrt{\frac{\left[M_{1}^{2}(E)-\left(M_{Y}+M_{\pi}\right)^{2}\right]\left[M_{1}^{2}(E)-\left(M_{Y}-M_{\pi}\right)^{2}\right]}{\left[M_{1}^{2}(0)-\left(M_{Y}+M_{\pi}\right)^{2}\right]\left[M_{1}^{2}(0)-\left(M_{Y}-M_{\pi}\right)^{2}\right]}} \\
& \times \Theta\left(M_{1}(E)-M_{Y}-M_{\pi}\right), \\
f_{2}^{Y}(E)= & \frac{M_{2}^{3}(0)}{M_{2}^{3}(E)} \sqrt{\frac{\left[M_{2}^{2}(E)-\left(M_{Y}+M_{N}\right)^{2}\right]\left[M_{2}^{2}(E)-\left(M_{Y}-M_{N}\right)^{2}\right]}{\left[M_{2}^{2}(0)-\left(M_{Y}+M_{N}\right)^{2}\right]\left[M_{2}^{2}(0)-\left(M_{Y}-M_{N}\right)^{2}\right]}} \\
& \times \Theta\left(M_{2}(E)-M_{Y}-M_{N}\right)
\end{aligned}
$$

with $M_{1}(E)=M_{\bar{K}}+M_{N}+E$ and $M_{2}(E)=M_{\bar{K}}+2 M_{N}+E$, where $M_{\bar{K}}, M_{N}, M_{Y}$ and $M_{\pi}$ denote the masses of $\bar{K}$, nucleon, hyperon $(Y=\Sigma$ or $\Lambda$ ), and $\pi$, respectively; $\Theta(x)=1$ for $x \geq 0$ and 0 for $x<0$. Fig. 1 displays these phase space suppression factors, as a function of $E$. $f_{1}^{\Sigma}(E)$ vanishes below the $\pi \Sigma N$ threshold of $E_{\mathrm{th}}(\pi \Sigma N)=-101 \mathrm{MeV}$, and $f_{1}^{\Lambda}(E)$ vanishes below the $\pi \Lambda N$ threshold of $E_{\mathrm{th}}(\pi \Lambda N)=-181 \mathrm{MeV} . f_{2}^{Y}(E)$ vanishes below the $Y N$ threshold, e.g., $E_{\text {th }}(\Sigma N)=-239 \mathrm{MeV}$ or $E_{\text {th }}(\Lambda N)=-319 \mathrm{MeV}$. As attempted in Refs. $[26,27]$, we take into account the energy dependence of the imaginary part multiplied by $f_{1}^{Y}(E)$ or $f_{2}^{Y}(E)$ in the optical potential. Thus we employ the energy-dependent $K^{-}$- " $p$ " optical potential which is parametrized in a Gaussian form with a range parameter $b$, as

$$
U^{\mathrm{opt}}(E ; \boldsymbol{r})=\left(V_{0}+i W_{0} f(E)\right) \exp \left[-(\boldsymbol{r} / b)^{2}\right]
$$

with

$$
f(E)=B_{1}^{(\pi \Sigma N)} f_{1}^{\Sigma}(E)+B_{1}^{(\pi \Lambda N)} f_{1}^{\Lambda}(E)+B_{2}^{(Y N)} f_{2}^{Y}(E),
$$

where $V_{0}$ and $W_{0}$ are adjusted parameters, of which values are determined to reproduce the result of the binding energy and width of the $K^{-} p p$ state in theoretical predictions or experimental data, as we will mention below. $B_{1}^{(\pi \Sigma N)}$ and $B_{1}^{(\pi \Lambda N)}$ are branching rates to $\left[K^{-} p p\right] \rightarrow \pi+\Sigma+N$ and $\left[K^{-} p p\right] \rightarrow \pi+\Lambda+N$ decay channels from the one-nucleon $K^{-}$ absorption, respectively, and $B_{2}^{(Y N)}$ is a branching rate to $\left[K^{-} p p\right] \rightarrow Y+N$ decay channel from the two-nucleon $K^{-}$absorption. Here we assumed [27, 41, 42]

$$
B_{1}^{(\pi \Sigma N)}=0.7, \quad B_{1}^{(\pi \Lambda N)}=0.1, \quad B_{2}^{(Y N)}=0.2,
$$

where we treat that the $\left[K^{-} p p\right] \rightarrow Y+N$ process acts effectively in $\Sigma+N$ and $\Lambda+N$ decay channels because these channels similarly affect the spectrum within the present framework (see also Fig. 1). Then we can rewrite the imaginary part of $U^{\text {opt }}(E)$ in Eq. (17) as

$$
\operatorname{Im} U^{\mathrm{opt}}(E ; \boldsymbol{r})=W_{1}^{\Sigma}(E ; \boldsymbol{r})+W_{1}^{\Lambda}(E ; \boldsymbol{r})+W_{2}^{Y}(E ; \boldsymbol{r}),
$$


where $W_{1}^{Y}(E ; \boldsymbol{r})$ and $W_{2}^{Y}(E ; \boldsymbol{r})$ correspond to the absorptive potentials for one- and twonucleon $K^{-}$absorptions, respectively:

$$
\begin{aligned}
& W_{1}^{Y}(E ; \boldsymbol{r})=B_{1}^{(\pi Y N)} W_{0} f_{1}^{Y}(E) \exp \left[-(\boldsymbol{r} / b)^{2}\right], \\
& W_{2}^{Y}(E ; \boldsymbol{r})=B_{2}^{(Y N)} W_{0} f_{2}^{Y}(E) \exp \left[-(\boldsymbol{r} / b)^{2}\right] .
\end{aligned}
$$

In the Green's function method, $S^{\text {con }}(E)$ of Eq. (10) can be further decomposed [23] as

$$
S^{\mathrm{con}}(E)=S_{\pi \Sigma N}^{\mathrm{con}}(E)+S_{\pi \Lambda N}^{\mathrm{con}}(E)+S_{Y N}^{\mathrm{con}}(E)
$$

with

$$
\begin{aligned}
S_{\pi Y N}^{\mathrm{con}}(E) & =-\frac{1}{\pi}\left\langle f^{\dagger} G^{\dagger}(E) W_{1}^{Y}(E) G(E) f\right\rangle, \\
S_{Y N}^{\mathrm{con}}(E) & =-\frac{1}{\pi}\left\langle f^{\dagger} G^{\dagger}(E) W_{2}^{Y}(E) G(E) f\right\rangle,
\end{aligned}
$$

where $S_{\pi Y N}^{\mathrm{con}}(E)$ and $S_{Y N}^{\mathrm{con}}(E)$ express components of the strength functions for the $\pi+Y+N$ decay process from the one-nucleon $K^{-}$absorption and for the $Y+N$ decay one from the two-nucleon $K^{-}$absorption, respectively, in the $K^{-}$conversion spectra. Therefore, the semi-exclusive spectra in the ${ }^{3} \mathrm{He}\left(\right.$ in-flight $\left.K^{-}, n\right)$ reaction,

$$
K^{-}+{ }^{3} \mathrm{He} \rightarrow n+\left[K^{-} p p\right] \rightarrow n+Y+X,
$$

can be evaluated in our calculations, where $Y=\{\Sigma, \Lambda\}$ and $X=\{\pi+N, N\}$.

\section{FIG. 2}

The binding energies B.E. and widths $\Gamma$ of the $K^{-} p p$ bound state with $I=1 / 2, J^{\pi}=0^{-}$ have been predicted in many calculations [5-12] and also reported in several experiments [15, 17, 18]. In Fig. 2, we summarize the values of B.E. and $\Gamma$ taken from theoretical predictions and experimental candidates. By considering these results of B.E. and $\Gamma$ as a guide, we attempt to construct the $K^{-}$- "pp" optical potentials $U^{\text {opt }}(E)$. We solve the Klein-Gordon equation self-consistently in the complex energy plane:

$$
\left\{\left(\omega(E)-V_{\text {Coul }}(\boldsymbol{r})\right)^{2}+\nabla^{2}-\mu^{2}-2 \mu U^{\mathrm{opt}}(E ; \boldsymbol{r})\right\} \Phi(E ; \boldsymbol{r})=0
$$

where $\Phi(E ; \boldsymbol{r})$ is a relative wave function between the $K^{-}$and " $p p$ " core nucleus, and $\omega(E)$ is a complex eigenvalue, as a function of $E$ which is a real number. If we find that $E$ satisfies

$$
\operatorname{Re} \omega(E)=E,
$$


we can obtain $\operatorname{Re} \omega(E)=-B . E$. and $\operatorname{Im} \omega(E)=-\Gamma / 2$ as the Klein-Gordon complex energy. Thus we determine the strength parameters of $\left(V_{0}, W_{0}\right)$ in Eq. (17) by fitting to the prediction or candidate of B.E. and $\Gamma$. Here we introduce four $K^{-}$- " $p p$ " optical potentials $U^{\text {opt }}(E)$ as follows:

(a) potential A which we determined by fitting to $B . E . \simeq 20 \mathrm{MeV}$ and the maximum $\Gamma \simeq$ $70 \mathrm{MeV}$ in a variational three-body calculation based on the Chiral unitary approach by Doté, Hyodo and Weise [7];

(b) potential $\mathrm{B}$ which is equivalent to the energy-independent optical potential obtained in a variational three-body calculation by Yamazaki and Akaishi [4], by fitting to B.E. = $48 \mathrm{MeV}$ and $\Gamma=61 \mathrm{MeV}$;

(c) potential $\mathrm{C}$ which we determined by fitting to $B . E . \simeq 70 \mathrm{MeV}$ and $\Gamma \simeq 110 \mathrm{MeV}$ in a $\bar{K} N N-\pi \Sigma N$ coupled-channel Faddeev calculation by Shevchenko, Gal and Mareš [5]. These values correspond to the maximum B.E. and $\Gamma$, respectively, within the uncertainty of their results;

(d) potential $\mathrm{D}$ which is a series of the potentials we determined by fitting to the experimental observations of B.E. and $\Gamma: \mathrm{D}_{1}, \mathrm{D}_{2}$ and $\mathrm{D}_{3}$ indicate the potentials for DISTO [18], FINUDA [15] and OBELIX [17] experiments, respectively.

For the range parameter for $U^{\mathrm{opt}}(E)$ in Eq. (17), here we used $b=1.09 \mathrm{fm}$, of which value is derived from the results of three-body calculations by Yamazaki and Akaishi [4]. A dependence of the spectrum on the range parameter $b$ is slightly seen in the QF region; for example, when $b$ is changed within $+0.12 \mathrm{fm}(-0.12 \mathrm{fm})$ in potential $\mathrm{C}$, the cross section of a QF peak is reduced (enhanced) by less than $10 \%$ and its peak position is shifted within -5 $\mathrm{MeV}(+5 \mathrm{MeV})$, while the bound-state spectrum is almost unchanged. Note that the values of $\Gamma$ which we considered in (a)-(c), are obtained by microscopic three-body calculations with only $\pi+Y+N$ decay processes in the one-nucleon $K^{-}$absorption $[4,5,7]$. Since a $K^{-}$- " $p p$ " optical potential has to describe not only one-nucleon $K^{-}$absorption processes but also two-nucleon ones, we employ the parameters of $\left(V_{0}, W_{0}\right)$ for potentials $\mathrm{A}, \mathrm{B}$ and $\mathrm{C}$ by fitting to the values of $B . E$. and $\Gamma$ without $B_{2}^{(Y N)}$ in Eq.(18), i.e., $\left(B_{1}^{(\pi \Sigma N)}, B_{1}^{(\pi \Lambda N)}, B_{2}^{(Y N)}\right.$ ) $=(0.7,0.1,0.0)$. For potentials $\mathrm{D}$, we took the parameters with $B_{2}^{(Y N)}$. In Table I, we list 
the parameter sets of $\left(V_{0}, W_{0}\right)$ for $U^{\text {opt }}(E)$, together with their calculated binding energies $B$.E and widths $\Gamma$ of the $K^{-} p p$ bound state. We find that when $B_{2}^{(Y N)}$ is switched on, the values of $B . E$. decrease and those of $\Gamma$ increase.

In Fig. 3, we display the real and imaginary parts of the $K^{-}-" p p$ " optical potentials $U^{\text {opt }}(E)$, as a function of a distance between the $K^{-}$and the center of the " $p p$ " core nucleus. If we neglect the energy-dependence in $U^{\mathrm{opt}}(E)$ of Eq. (17) by replacing $f(E)$ by 1 , we find the energy-independent optical potentials $U_{0}^{\text {opt }}$, as used in our previous calculation [23]. It should be noticed the values of $W_{0}$ in $U_{0}^{\text {opt }}$ differ from those in $U^{\text {opt }}(E)$, as shown in Table I, whereas we have $\operatorname{Im} U_{0}^{\mathrm{opt}}=\operatorname{Im} U^{\mathrm{opt}}(E)$ at $E=-B$.E.

\section{TABLE I}

\section{FIG. 3}

\section{NUMERICAL RESULTS}

\section{A. Inclusive spectrum by the ${ }^{3} \mathrm{He}\left(\mathrm{in}-\mathrm{flight} K^{-}, n\right)$ reaction}

Let us consider the ${ }^{3} \mathrm{He}\left(\right.$ in-flight $\left.K^{-}, n\right)$ reaction at $p_{K^{-}}=1.0 \mathrm{GeV} / \mathrm{c}$ and $\theta_{\text {lab }}=0^{\circ}$ for the J-PARC E15 experiment [19]. To find possible evidence of the $K^{-} p p$ bound state, we evaluate the inclusive and semi-exclusive spectra of the ${ }^{3} \mathrm{He}\left(\right.$ in-flight $\left.K^{-}, n\right)$ reaction numerically by Eqs. (3), (9)-(11) and (23)-(25).

FIG. 4

In Fig. 4, we display the calculated results of the inclusive spectra with the optical potentials $U^{\text {opt }}(E)$ listed in Table I. In Fig. 4(c), we show the calculated inclusive spectrum for potential $\mathrm{C}$ where the binding energy and width of the $K^{-} p p$ bound state are obtained as $B . E .=59 \mathrm{MeV}$ and $\Gamma=164 \mathrm{MeV}$, respectively. The inclusive spectrum with $U^{\mathrm{opt}}(E)$ is qualitatively different from that with the energy-independent $U_{0}^{\text {opt }}$. The former has a cusp which appears at the $\pi \Sigma N$ threshold in the $L=0$ component of the spectrum, whereas the latter has no peak due to its large width of the $K^{-} p p$ state [23]. Such a cusp-like structure originates from the energy dependence of the imaginary part of $U^{\mathrm{opt}}(E)$, and its mechanism can be understood by behavior of a pole trajectory of the $K^{-} p p$ state in the complex energy plane, as we will discuss in Sect. III C. 
In Fig. 4(b), we show the inclusive spectrum for potential B which gives B.E. $=45 \mathrm{MeV}$ and $\Gamma=82 \mathrm{MeV}$. A clear peak of the $K^{-} p p$ state appears in both the spectra with $U^{\text {opt }}(E)$ and $U_{0}^{\text {opt }}$, but the peak position for $U^{\text {opt }}(E)$ is slightly shifted from $E=-45 \mathrm{MeV}$ to -50 $\mathrm{MeV}$ because of its energy dependence. Since this state is away from the branching point of the $\pi \Sigma N$ threshold, the peak in the spectrum is scarcely influenced by the threshold, so that its shape approximately indicates a standard Breit-Wigner (BW) form [28]. Since a $\pi \Sigma N$ phase space is taken into account, the spectrum for $U^{\text {opt }}(E)$ is suppressed below the $\pi \Sigma N$ decay threshold of $E_{\mathrm{th}}(\pi \Sigma N) \simeq-100 \mathrm{MeV}$, in contrast to that for $U_{0}^{\text {opt }}[23]$.

In Fig. 4(a), we find that a peak of the $K^{-} p p$ bound state is not clear in the inclusive spectrum with potential A, because of the relatively small binding energy of $15 \mathrm{MeV}$ with the large width of $92 \mathrm{MeV}$. The energy dependence of the spectrum for $U^{\text {opt }}(E)$ seems to be similar to that of potential B. Yamagata et al. [11] also performed a similar calculation using the energy-dependent $K^{-}$- " $p p$ " optical potential based on the Chiral unitary model. The shape of their inclusive spectrum is different from that of ours because of a different contribution of the partial-wave components and their different widths; they found a $K^{-} p p$ $L=0$ bound state with $B . E . \simeq 20 \mathrm{MeV}$ and $\Gamma \simeq 40 \mathrm{MeV}$, and other $L=1,2$ bound states with $B . E . \simeq 10 \mathrm{MeV}[11]$. However, it is not understood that the $K^{-} p p$ system has nuclear bound states with $L \geq 1$, in our consideration to its small nuclear size such as ${ }^{3} \mathrm{He}$ [43].

We consider potential $\mathrm{D}_{2}$ as a typical example of the potential $\mathrm{D}$ series. In Fig. 4(d), we show the inclusive spectrum with potential $\mathrm{D}_{2}$ by fits to the experimental values, B.E. = $115 \mathrm{MeV}$ and $\Gamma=67 \mathrm{MeV}$, which are obtained from the invariant-mass spectrum by the FINUDA experiment [15]. If an interpretation of the FINIDA candidate as a $K^{-} p p$ bound state is true, a clear peak should appear below the $\pi \Sigma N$ threshold in the missing-mass spectrum. It would be easy to observe such a peak structure experimentally. The spectra with $U^{\text {opt }}(E)$ and $U_{0}^{\text {opt }}$ are quite similar in shape below the $\pi \Sigma N$ threshold, whereas they are considerably different each other above the $\pi \Sigma N$ threshold. For potentials $\mathrm{D}_{1}$ and $\mathrm{D}_{3}$ which are determined respectively by fits to the data of DISTO [18] and OBELIX [17], we also obtain that a clear peak in their spectra appears below the $\pi \Sigma N$ threshold (see also Fig. 10). We find that the shape of the peak in the spectrum for $D_{1}$ is similar to that for $\mathrm{D}_{2}$, as shown in Fig. $4(\mathrm{~d})$, whereas the spectrum for $\mathrm{D}_{3}$ has a very prominent peak because of the small width of $\Gamma=24 \mathrm{MeV}$. 
Therefore, we recognize that the inclusive spectrum of the ${ }^{3} \mathrm{He}\left(\right.$ in-flight $\left.K^{-}, n\right)$ reaction at $p_{K^{-}}=1.0 \mathrm{GeV} / \mathrm{c}$ and $\theta_{\text {lab }}=0^{\circ}$ is expected to have a signal for clear evidence of the $K^{-} p p$ bound state. The calculated spectrum predominately has a bound state with $I=1 / 2, J^{\pi}=$ $0^{-}$and an orbital angular momentum $L=0$ in the $K^{-} p p$ bound region below the $K^{-}+p+p$ threshold, whereas continuum states with $L \geq 1$ occur in the QF region. We stress that the (in-flight $K^{-}, n$ ) reaction on the $s$-shell nuclear target such as ${ }^{3} \mathrm{He}$ provides an advantage to produce the deeply-bound $K^{-}$nuclear state with $L=0$. These results also indicate the importance of the energy-dependence in the $K^{-}-_{-} " p p$ " optical potentials, particularly, in the case of potentials $\mathrm{C}, \mathrm{D}_{1}$ and $\mathrm{D}_{2}$. We realize the limit to apply the energy-independent optical potential to calculations for the spectrum; such a potential can be justified only for potential A involving the shallow potentials [7, 11], and it works approximately for potential B around the $K^{-} p p$ peak. The strength of $\left|V_{0}\right|$ in $U_{0}^{\text {opt }}$ must be shallower than $300 \mathrm{MeV}$, which corresponds to B.E. $<50 \mathrm{MeV}$.

\section{B. Contribution of one- and two-nucleon $K^{-}$absorption processes in the $K^{-}$con-} version spectra

\section{FIG. 5}

It should be noticed that the calculated $K^{-}$conversion spectra can be directly compared with the experimental data at the J-PARC E15 experiment, which is planned to measure the (in-flight $K^{-}, n$ ) spectra and the decaying particles from the $K^{-} p p$ system simultaneously [19]; no $K^{-}$escape spectrum will be measured in this experiment. To search a better signal for the $K^{-} p p$ bound state, we focus on the $K^{-}$conversion spectra which express semiexclusive $K^{-}+{ }^{3} \mathrm{He} \rightarrow n+Y+X$ processes in the deeply-bound region, where $Y=\{\Sigma, \Lambda\}$ and $X=\{\pi+N, N\}$. By Eqs. (24) and (25), we can calculate their strength functions for $K^{-}$conversion processes, which are effectively described by the imaginary potential, $\operatorname{Im} U^{\text {opt }}(E)$ in Eq. (20), within the optical models. For the $K^{-}$escape processes, we can calculate the strength function in Eq. (11), which is probably underestimated because it has be described as $K^{-}+$" $p p$ " continuum states above the $K^{-}+p p$ threshold where " $p p$ " should actually break up into $p+p$.

In Fig. 5, we illustrate partial contributions of the ${ }^{3} \mathrm{He}\left(\right.$ in-flight $\left.K^{-}, n\right)$ spectrum at $p_{K^{-}}=$ $1.0 \mathrm{GeV} / \mathrm{c}$ and $\theta_{\text {lab }}=0^{\circ}$, e.g., $\left[K^{-} p p\right] \rightarrow \pi+\Sigma+N$ decay process and $\left[K^{-} p p\right] \rightarrow \pi+\Lambda+N$ 
decay process from the one-nucleon $K^{-}$absorption, and $\left[K^{-} p p\right] \rightarrow Y+N$ decay process from the two-nucleon $K^{-}$absorption. In Fig. 5(c), we find that a cusp-like peak at $\pi \Sigma N$ threshold originates from the $\pi+\Lambda+N$ and $Y+N$ decay channels in the spectrum for potential $\mathrm{C}$, rather than the $\pi+\Sigma+N$ decay one which is suppressed below the $\pi \Sigma N$ threshold because its decay channel is closed there. Such a cusp-like peak is clear evidence of the formation of the $K^{-} p p$ bound state. Since the observed peak position and width do not directly correspond to the actual binding energy and width, respectively, we need a comparison between the theoretical and experimental spectra to extract the binding energy and width of the $K^{-} p p$ bound state from the cusp-like peak.

In Fig. 5(d), it is also interesting to see a clear peak in the $\pi+\Lambda+N$ and $Y+N$ decay spectra for potential $\mathrm{D}_{2}$ which is more attractive than potential C. Its shape is asymmetric because it must be sharply cut by the phase space suppression factor above the $\pi \Sigma N$ threshold. Moreover, we confirm that the $\pi+\Sigma+N$ decay spectrum gives no peak for the $K^{-} p p$ bound state, as well as the spectrum for potential C.

In Fig. 5(b), we show partial contributions of the calculated spectrum with potential B. We find that the shape of the spectra with $\pi+\Sigma+N, \pi+\Lambda+N$ and $Y+N$ decay processes are essentially the same, but the peak positions of their spectra are slightly different each other because of the energy dependence of the potential. A clear signal would be observed in the spectrum with $\left[K^{-} p p\right] \rightarrow Y+N$ from the two-nucleon $K^{-}$absorption, as well as the inclusive spectrum shown in Fig. 4(b).

On the other hand, we confirm that there is no peak in any partial contributions with potential A even if the $K^{-} p p$ bound state exists, as shown in Fig. 5(a). This state exists close to the $K^{-}+p+p$ threshold due to a small binding energy of B.E. $=15 \mathrm{MeV}$ and a large width of $\Gamma=92 \mathrm{MeV}$. For a more quantitative estimation, we need to examine a whole shape of the spectrum including the effects of the $K^{-}+p+p$ threshold [24, 28].

We recognize that the detailed comparison between the theoretical and experimental spectra is required to extract the binding energy and width of the $K^{-} p p$ bound state from the spectra. The shape behavior of the $\left[K^{-} p p\right] \rightarrow \pi+\Lambda+N$ decay spectrum is quite similar to that of the $\left[K^{-} p p\right] \rightarrow Y+N$ one in all of our potentials. This similarity is understood from the fact that the energy dependence of the phase space factor $f_{1}^{\Lambda}(E)$ for the $\pi+\Lambda+N$ decay processes resembles that of $f_{2}^{Y}(E)$ for the $Y+N$ decay processes near the $\pi \Sigma N$ threshold. 


\section{Pole trajectory for the deeply-bound $K^{-} p p$ state}

1. Moving pole in the complex energy plane

FIG. 6

It is important to understand the mechanism of a peak structure near the $\pi \Sigma N$ threshold in the spectrum, so as to identify the nature of the $K^{-} p p$ bound state from the experimental data. Quantum mechanically, the peak structure in the energy spectrum is associated with a pole in the scattering amplitude or the complete Green's function. The pole position corresponds to a complex eigenvalue of a Hamiltonian on the complex energy plane. The shape of the spectrum must be modified by the threshold effects if the pole is located near the branch point of the threshold. To understand the shape behavior of the $\left[K^{-} p p\right] \rightarrow Y+N$ decay spectrum, we investigate the pole position of the $K^{-} p p$ state in the complex energy plane. We can obtain the pole position as a complex eigenvalue of $\omega(E)$ in Eq. (27) as a function of $E$ because of the energy dependence of $U^{\text {opt }}(E)$.

The shape of the inclusive spectrum in the $K^{-}$bound region is perhaps written as the following form:

$$
S^{(\text {pole })}(E)=-\frac{1}{\pi} \frac{\operatorname{Im} \omega(E)}{D^{2}(E)},
$$

where

$$
D(E) \equiv \sqrt{(E-\operatorname{Re} \omega(E))^{2}+(\operatorname{Im} \omega(E))^{2}}
$$

denotes a distance between a point $(E, 0)$ of the physical state on the real axis and the pole at a point $(\operatorname{Re} \omega(E), \operatorname{Im} \omega(E))$ in the complex energy plane, as illustrated in Fig. 6. If the energy dependence of $\omega(E)$ is negligible, the shape of $S^{(\text {pole })}(E)$ is equivalent to the BW resonance form. Since $\operatorname{Im} \omega(E)$ is approximately proportional to the phase space suppression factor $f(E)$, the shape of the inclusive spectrum is roughly denoted by $f(E) / D^{2}(E)$. Functions of $f_{1}^{Y}(E) / D^{2}(E)$ and $f_{2}^{Y}(E) / D^{2}(E)$ can simulate the shapes of the one- and two-nucleon $K^{-}$absorption spectra, respectively. It is apparent that $S_{\pi \Sigma N}^{\text {con }}(E)$ is suppressed below the $\pi \Sigma N$ threshold due to the behavior of the function $f_{1}^{Y}(E)$. On the other hand, $S_{\pi \Lambda N}^{\mathrm{con}}(E)$ and $S_{Y N}^{\text {con }}(E)$ are approximately equivalent to $1 / D^{2}(E)$ because $f_{1}^{\Lambda}(E)$ and $f_{2}^{Y}(E)$ can be regarded as a constant around the $\pi \Sigma N$ threshold. For instance, we have

$$
S_{Y N}^{\mathrm{con}}(E) \approx \text { const. } \times \frac{1}{D^{2}(E)}
$$


for the $Y+N$ decay spectrum.

FIG. 7

Now we consider the peak structure in the $\left[K^{-} p p\right] \rightarrow Y+N$ decay spectrum obtained with $V_{0}=-292 \mathrm{MeV}$ and $W_{0}=-107 \mathrm{MeV}$ for potential B. In Fig. 7, we illustrate its pole trajectory of $\omega(E)$ as a function of $E$, together with $D(E)$ and $1 / D^{2}(E)$. When $E$ is changed from $0 \mathrm{MeV}$ to $-100 \mathrm{MeV}$, the pole moves slowly from a point $(-42 \mathrm{MeV},-51$ $\mathrm{MeV})$ to a point $(-51 \mathrm{MeV},-15 \mathrm{MeV})$, so that $D(E)$ works in an almost smooth function with the minimum value at $E \simeq-60 \mathrm{MeV}$. In this case, therefore, a clear peak in $1 / D^{2}(E)$ is observed around $E \simeq-60 \mathrm{MeV}$. This peak is in good agreement with that of the $Y+N$ decay spectrum in Fig. 5(b). The shape of the spectrum is deviated from the standard BW form due to the nature of the energy dependence of $D(E)$, whereas the position of this peak does not coincide with a point at $E=-B . E .=-45 \mathrm{MeV}$.

\section{FIG. 8}

\section{FIG. 9}

In Fig. 5(c), on the other hand, we have shown the cusp-like peak at the $\pi \Sigma N$ threshold in the $\left[K^{-} p p\right] \rightarrow Y+N$ decay spectrum for potential C. For understanding the appearance of such a cusp-like structure, we obtain a moving pole at $\omega(E)$ with $V_{0}=-344 \mathrm{MeV}$ and $W_{0}=-203 \mathrm{MeV}$ for potential $\mathrm{C}$, as a function of $E$. In Fig. 8, we illustrate the pole trajectory of their $\omega(E), D(E)$ and $1 / D^{2}(E)$. We find that the pole of $\omega(E)$ moves widely in the complex energy plane; when $E$ is changed from $0 \mathrm{MeV}$ to $-100 \mathrm{MeV}$, the pole of $\omega(E)$ moves from a point $(-43 \mathrm{MeV},-110 \mathrm{MeV})$ to a point $(-76 \mathrm{MeV},-34 \mathrm{MeV})$. For $E<E_{\mathrm{th}}(\pi \Sigma N)$, its pole remains around the point $(-77 \mathrm{MeV},-25 \mathrm{MeV})$. It should be noticed that the minimum of $D(E)$ is realized at $E=E_{\mathrm{th}}(\pi \Sigma N)$ where $d D(E) / d E$ is singular. In this case, therefore, a cusp-like peak in $1 / D^{2}(E)$ is observed at the $\pi \Sigma N$ threshold. This shape agrees with that of the spectrum shown in Fig. 5(c). In order to see the effects of $W_{0}$, we also obtain a trajectory of the moving pole with $V_{0}=-344 \mathrm{MeV}$ and $W_{0}=-47 \mathrm{MeV}$, which corresponds to a specific case with an artificial narrow width. We confirm that the shape of its spectrum is identified as the BW form, as shown in Fig. 9.

Consequently, we recognize that the cusp-like structure can be described as behavior of the pole trajectory which is governed by the energy dependence of $U^{\text {opt }}(E)$, as well as a clear 
peak with the BW form. The path of the trajectory for the moving pole in the complex energy plane is determined by the values of $V_{0}$, and its moving range on the trajectory depends on the values of $W_{0}$.

2. Pole trajectories by the $K^{-}$- "pp" optical potentials

FIG. 10

In Fig. 10, we show the pole trajectories of the $K^{-} p p$ bound state for potentials $\mathrm{A}, \mathrm{B}, \mathrm{C}$, $\mathrm{D}_{1}, \mathrm{D}_{2}$ and $\mathrm{D}_{3}$ in the complex energy plane. The strength parameters of $\left(V_{0}, W_{0}\right)$ characterize the shape structure of the $K^{-} p p$ state in the spectrum with the $\left[K^{-} p p\right] \rightarrow Y+N$ decay from the two-nucleon $K^{-}$absorption. For potential C, a cusp at the $\pi \Sigma N$ threshold appears clearly in the spectrum, as seen in Fig. $5(\mathrm{c})$, because the value of $D(E)$ at $E=E_{\mathrm{th}}(\pi \Sigma N)$ is much smaller than that of $\Gamma / 2$ which is equivalent to the distance from the pole at a point $(-B . E .,-\Gamma / 2)$ to the real axis. For potential $\mathrm{D}_{2}$, a steep step is observed at the $\pi \Sigma N$ threshold, as seen in Fig. 5(d); its yield is sharply cut down because its pole is rapidly moving above the $\pi \Sigma N$ threshold.

\section{FIG. 11}

One should be noticed that our $K^{-}-_{-} " p p$ " optical potentials, $U^{\text {opt }}(E)$, are not derived from microscopic calculations, but are introduced phenomenologically. To examine whether the potential has the appropriate energy dependence or not, we evaluate the pole trajectory of a point $(-B . E .,-\Gamma / 2)$ in the complex energy plane when we change the value of $V_{0}$ in $U^{\text {opt }}(E)$.

In Fig. 11, we show the energy dependence of the pole trajectories on decay channels with $V_{0}=(-300)-(-420) \mathrm{MeV}$, when we switch on/off each $B_{1}^{(\pi \Lambda N)}$ and $B_{2}^{(Y N)}$ with $B_{1}^{(\pi \Sigma N)}=0.7$ and $W_{0}=-203 \mathrm{MeV}$ which corresponds to the imaginary part of the potential $\mathrm{C}$. If we consider only $B_{1}^{(\pi \Sigma N)}$, its width becomes smaller when the $-B . E$. is close to $E_{\mathrm{th}}(\pi \Sigma N)$, and it finally becomes 0 when $-B$.E. is located below $E_{\mathrm{th}}(\pi \Sigma N)$. This behavior seems to be qualitatively consistent with the result obtained from a $\bar{K} N N-\pi \Sigma N$ coupled-channel Faddeev calculation by Ikeda and Sato [44]. Even if $B_{1}^{(\pi \Lambda N)}$ and/or $B_{2}^{(Y N)}$ are switched on, the pole trajectory of the point $(-B . E .,-\Gamma / 2)$ is not so changed quantitatively, except for an additional width. But the pole trajectory for the energy-independent potential $U_{0}^{\text {opt }}$ differs 
from that for $U^{\text {opt }}(E) ;-\Gamma / 2$ is almost proportional to $-B$. E. Therefore, we believe that our $K^{-}$- "pp" optical potential $U^{\text {opt }}(E)$ has the desirable energy dependence which is expected from the coupled-channel Faddeev calculation, and that it is enough for us to discuss the shape of the spectrum with the $\left[K^{-} p p\right] \rightarrow Y+N$ decay process. For more quantitative argument, one should make the $\bar{K} N N$ single-channel effective potential, in which the $\pi \Sigma N$ channel is eliminated in the $\bar{K} N N-\pi \Sigma N$ coupled channel scheme [45], and compare it with our optical potential. The investigation along this line will be discussed in future works.

\section{DISCUSSIONS}

\section{A. Cusp-like structure in the spectrum near the $\pi \Sigma N$ threshold}

Recently, Akaishi et al. [46] have discussed a cusp-like structure in the spectrum of the (inflight $K^{-}, n$ ) reaction on a deuteron target, using a coupled-channel model with a separable potential. They have shown that the cusp-like structure at the $\pi \Sigma$ threshold can be also observed in the $\left[K^{-} p\right] \rightarrow \pi+\Sigma$ spectrum from the one-nucleon $K^{-}$absorption. In Sect. III, we have found a cusp-like structure at the $\pi \Sigma N$ threshold in the spectra with $\left[K^{-} p p\right] \rightarrow$ $\pi+\Lambda+N$ and $\left[K^{-} p p\right] \rightarrow Y+N$ decay precesses if we use potential C. The shape and magnitude of these spectra strongly depend on the pole trajectory of the $K^{-} p p$ bound state, and are characterized by the strength parameters of $\left(V_{0}, W_{0}\right)$. It is worth examining the condition for $\left(V_{0}, W_{0}\right)$ which gives the cusp-like structure at the $\pi \Sigma N$ threshold within our optical potential, $U^{\mathrm{opt}}(E)$.

In this subsection, we focus on the spectra with $\left[K^{-} p p\right] \rightarrow \pi+\Sigma+N$ and $\left[K^{-} p p\right] \rightarrow Y+N$ decays, by artificially changing $\left(V_{0}, W_{0}\right)$ in the following two cases: (i) $\left(B_{1}^{(\pi \Sigma N)}, B_{2}^{(Y N)}\right)=$ $(0.7,0.0)$ which means only the $\left[K^{-} p p\right] \rightarrow \pi+\Sigma+N$ decay process; (ii) $\left(B_{1}^{(\pi \Sigma N)}, B_{2}^{(Y N)}\right)=$ $(0.7,0.2)$. Here we omit $B_{1}^{(\pi \Lambda N)}$ for the $\left[K^{-} p p\right] \rightarrow \pi+\Lambda+N$ decay process for simplicity, because $B_{1}^{(\pi \Lambda N)}$ operates similarly to $B_{2}^{(Y N)}$ in the spectrum, as discussed in Sect. III B.

\section{FIG.12}

In Fig. 12, we show the behaviors of $S(E)$ in the spectra with $\left[K^{-} p p\right] \rightarrow \pi+\Sigma+N$ and $\left[K^{-} p p\right] \rightarrow Y+N$ decays at $-W_{0}=107 \mathrm{MeV}$, which corresponds to the imaginary part of potential B, by changing $-V_{0}=340-400 \mathrm{MeV}$. For the case of (i), as seen in Fig. 12(left), the 
magnitude of the peak in $S_{\pi \Sigma N}^{\text {con }}(E)$ grows at the $\pi \Sigma N$ threshold as increasing $-V_{0}$. When $-V_{0} \simeq 380 \mathrm{MeV}$, the magnitude is at its maximum around $-B . E . \simeq E_{\mathrm{th}}(\pi \Sigma N)$. When $-V_{0}>380 \mathrm{MeV}$, the $K^{-} p p$ state must be bound below the $\pi \Sigma N$ threshold and its peak is located at $E=-B$. E. Such a cusp-like peak in $S_{\pi \Sigma N}^{\mathrm{con}}(E)$ is quite similar to that obtained by Akaishi et al. [46].

For the case of (ii), we show the behaviors of $S_{Y N}^{\text {con }}(E)$ and $S_{\pi \Sigma N}^{\text {con }}(E)$ with $B_{2}^{(Y N)}=0.2$ in Fig. 12(right). We find that there is the cusp-like structure in $S_{Y N}^{\text {con }}(E)$ at $-V_{0} \simeq 340-380$ $\mathrm{MeV}$, and the asymmetric peak which is cut off sharply above the $\pi \Sigma N$ threshold appears in $S_{Y N}^{\text {con }}(E)$ when $-V_{0}>380 \mathrm{MeV}$, whereas there is no (cusp-like) peak in $S_{\pi \Sigma N}^{\text {con }}(E)$. Thus, we recognize that the cusp-like structure is observed in the $\left[K^{-} p p\right] \rightarrow Y+N$ decay spectrum rather than the $\left[K^{-} p p\right] \rightarrow \pi+\Sigma+N$ decay one because of the existence of the $Y+N$ decay channel.

FIG. 13

In Fig. 13, we examine the behaviors of $S(E)$ in the spectra with $\left[K^{-} p p\right] \rightarrow \pi+\Sigma+N$ and $\left[K^{-} p p\right] \rightarrow Y+N$ decays at $-V_{0}=344 \mathrm{MeV}$, which corresponds to the real part of potential C, by changing $-W_{0}=60-200 \mathrm{MeV}$. In the case of (i), we obtain that a clear peak near $E \simeq$ $-80 \mathrm{MeV}$ in $S_{\pi \Sigma N}^{\mathrm{con}}(E)$ becomes broad as $-W_{0}$ increases, as shown in Fig. 13(left). In the case of (ii), we also find that a clear peak is located near $E \simeq-80 \mathrm{MeV}$ in both of $S_{\pi \Sigma N}^{\text {con }}(E)$ and $S_{Y N}^{\text {con }}(E)$ at $-W_{0}=60 \mathrm{MeV}$, as shown in Fig. 13(right). As increasing $-W_{0}, S_{\pi \Sigma N}^{\text {con }}(E)$ is gradually reduced, but $S_{Y N}^{\text {con }}(E)$ is gradually enhanced just at the $\pi \Sigma N$ threshold. Thus it grows up a threshold-cusp in the spectrum.

Therefore, we have the cusp-like structure in the spectrum under the conditions that $-B$.E. is close to and above the $\pi \Sigma N$ threshold energy and $\Gamma$ is considerably large. The corresponding strength parameters are roughly estimated as $-V_{0}=330-380 \mathrm{MeV}$ and $-W_{0} \geq$ 100-120 MeV, which are found in such as potential C given in Table I. The cusp-like structure is the unique signal for evidence of the deeply bound $K^{-} p p$ state in the $\left[K^{-} p p\right] \rightarrow Y+N$ decay spectrum. 


\section{B. Dependence of the spectrum on the branching rate of $B_{2}^{(Y N)}$}

The shape of the semi-exclusive $K^{-}$conversion spectrum including the $\left[K^{-} p p\right] \rightarrow Y+$ $N$ decay process is very important to extract the structure of the $K^{-} p p$ state, e.g., the potential strengths of $\left(V_{0}, W_{0}\right)$, rather than that of the spectrum including the $\left[K^{-} p p\right] \rightarrow$ $\pi+\Sigma+N$ decay process. In our calculations, we assumed the branching rate of $B_{2}^{(Y N)}=$ 0.2 in the $K^{-} p p$ decay processes. This value is often used in previous works for heavier targets $[26,27,41,42]$ but it is experimentally unknown in the $K^{-}$absorption on ${ }^{3} \mathrm{He}$ in flight. In terms of the $K^{-}$absorption on ${ }^{4} \mathrm{He}$ at rest, the early data of the helium bubble chamber experiment [47] suggested that the ratio of the two-nucleon $K^{-}$absorption to all the $K^{-}$absorption processes amounts to $16 \%$, whereas its value depends on atomic orbits where $K^{-}$is absorbed through atomic cascade processes [27, 48]. A recent analysis of the $K^{-}$absorption on ${ }^{4} \mathrm{He}$ at rest [49] also calls for reexamination of $B_{2}^{(Y N)}$ experimentally. To

determine the value of $B_{2}^{(Y N)}$ in the $K^{-}$absorption in flight, we need more investigations on $B_{2}^{(Y N)}$ experimentally and theoretically.

\section{FIG. 14}

As the first step forward the investigations, we attempt to calculate the strength function $S(E)$ in potential $\mathrm{C}$, in order to check the sensitivity of semi-exclusive spectra on the value of $B_{2}^{(Y N)}$. In Fig. 14, we demonstrate the dependence of the spectra on the values of $B_{2}^{(Y N)}$ when changing $B_{2}^{(Y N)}=0.1-0.3$. For the $\left[K^{-} p p\right] \rightarrow \pi+\Sigma+N$ and $\left[K^{-} p p\right] \rightarrow \pi+\Lambda+N$ decay spectra, each magnitude is reduced as $B_{2}^{(Y N)}$ increases, as shown in Figs. 14(a) and (b). On the other hand, the $\left[K^{-} p p\right] \rightarrow Y+N$ decay spectrum is enhanced as $B_{2}^{(Y N)}$ increases, as shown in Fig. 14(c). The shape of these spectra is scarcely modified by a small change of $B_{2}^{(Y N)}$. Thus, the detailed values of the branching rates have an influence only on the relative magnitude of each decay spectrum, without changing the nature of the $K^{-} p p$ formation signal. We stress that it is important to compare the shapes of the calculated spectra with those of the measured ones. This detailed comparison provides valuable information on $B_{2}^{(\pi \Sigma N)}, B_{2}^{(\pi \Lambda N)}$ and $B_{2}^{(Y N)}$, as well as on the binding energy and width of the $K^{-} p p$ state. 


\section{The spectrum near the $K^{-}+p+p$ threshold}

In Fig. 5(a), we have shown partial contributions of the spectra with potential A. The spectra have no clear peak because the pole for potential A has a large width and is located near the $K^{-}+p+p$ threshold. In order to extract information on the $K^{-}$" $p p$ " potential from their spectral shape, one needs to consider the effect of the $K^{-}+p+p$ threshold beyond the " $p p$ " core assumption. If " $p p$ " $\rightarrow p+p$ degree of freedom is taken into account, a QF $\Lambda(1405)$ formation via $\left[K^{-} p p\right] \rightarrow$ " $K^{-} p "+p \rightarrow \Lambda(1405)+p$ would be important rather than $\left[K^{-} p p\right] \rightarrow K^{-}+p+p$ break-up processes. The spectrum of such a QF $\Lambda(1405)$ formation stands up from $E \simeq(-10)-(-20) \mathrm{MeV}$ below the $K^{-}+p+p$ threshold, which depends on the $\Lambda(1405)$ mass as a $\bar{K}-N$ quasibound state. In the case of $B . E . \simeq 20 \mathrm{MeV}$ obtained in potential A, therefore, it may be necessary to estimate the contribution of the QF $\Lambda(1405)$ spectrum which contaminates into the $K^{-} p p$ formation spectrum near the $K^{-}+p+p$ threshold.

\section{SUMMARY AND CONCLUSION}

We have examined the inclusive and semi-exclusive spectra in the ${ }^{3} \mathrm{He}\left(\right.$ in-flight $\left.K^{-}, n\right)$ reaction at $p_{K^{-}}=1.0 \mathrm{GeV} / \mathrm{c}$ and $\theta_{\text {lab }}=0^{\circ}$ for the forthcoming J-PARC E15 experiment. We have discussed these spectra with the energy-dependent $K^{--_{-}}$" $p p$ " optical potentials $U^{\text {opt }}(E)$, based on the results of the binding energies and widths of the $K^{-} p p$ (unstable) bound states in several predictions or candidates. To understand the peak structure in the spectrum, we have investigated the trajectory of the moving pole of the $K^{-} p p$ bound state in the complex energy plane, and the behavior of the corresponding strength function by changing the strength parameters $\left(V_{0}, W_{0}\right)$ of $U^{\mathrm{opt}}(E)$ systematically. The calculated spectrum predominately has the bound state with $I=1 / 2, J^{\pi}=0^{-}$and $L=0$ in the $K^{-} p p$ bound region below the $K^{-}+p+p$ threshold, whereas the continuum states with $L \geq 1$ occur in the QF region. We have shown that the (in-flight $K^{-}, n$ ) reaction on the $s$-shell nuclear targets such as ${ }^{3}$ He provides an advantage to produce the deeply-bound $K^{-}$nuclear state with $L=0$. The results are summarized as follows:

(i) The clear peak appears below the $\pi \Sigma N$ threshold in the spectrum with the $\left[K^{-} p p\right] \rightarrow$ $Y+N$ decay from the two-nucleon $K^{-}$absorption as evidence of the $K^{-} p p$ bound 
state, within $-V_{0}>380 \mathrm{MeV}$, as the case of potentials D.

(ii) The cusp-like structure appears at the $\pi \Sigma N$ threshold in the $\left[K^{-} p p\right] \rightarrow Y+N$ decay spectrum within $-V_{0} \simeq 330-380 \mathrm{MeV}$ and $-W_{0}>\sim 110 \mathrm{MeV}$, rather than in the $\left[K^{-} p p\right] \rightarrow \pi+\Sigma+N$ decay spectrum, as the case of potential C.

(iii) The distinct peak in the $\left[K^{-} p p\right] \rightarrow Y+N$ and $\left[K^{-} p p\right] \rightarrow \pi+\Sigma+N$ decay spectra is observed as clear evidence of the $K^{-} p p$ bound state within $-V_{0} \simeq 200-330 \mathrm{MeV}$ and $-W_{0}<\sim 110 \mathrm{MeV}$ such as potential B, whereas no clear peak is observed in these spectra even if the $K^{-} p p$ bound state exists within $-V_{0} \simeq 200-330 \mathrm{MeV}$ and $-W_{0}>$ $\sim 110 \mathrm{MeV}$ such as potential A.

In conclusion, the ${ }^{3} \mathrm{He}\left(\right.$ in-flight $\left.K^{-}, n\right)$ spectrum including the $\left[K^{-} p p\right] \rightarrow Y+N$ decay process from the two-nucleon $K^{-}$absorption provides evidence of the $K^{-} p p$ bound state to identify itself as the appropriate $K^{-}{ }^{-}$" $p p$ " potential with the help of the trajectory of its moving pole in the complex energy plane. If any of the experimental observations of DISTO [18], FINUDA [15] and OBELIX [17] indicates evidence of the $K^{-} p p$ bound state, its corresponding peak should appears below the $\pi \Sigma N$ threshold in the J-PARC E15 spectrum. Otherwise, we will realize that these experimental data are all incorrect. Moreover, the cusp-like structure is the unique signal of the $K^{-} p p$ formation, as well as the peak structure. This phenomenology suggests the possibility of observing the cusp-like structure obtained by the deep potential with strong absorption $\left(-V_{0}=330-380 \mathrm{MeV},-W_{0}>110 \mathrm{MeV}\right)$, as predicted by Shevchenko et al. [5]. If a cusp-like structure is observed, a precise comparison between theoretical and experimental spectra is required to extract the binding energy and width of the $K^{-} p p$ state, as well as the analysis of the spectrum in which the clear peak is observed. To get more quantitative results on the cusp-like or peak structure, a full microscopic calculation between $\bar{K} N N$ and $\pi Y N$ channels would be required beyond our optical potential models. Nevertheless, we believe that our calculations lead to a good insight for qualitative understanding the spectrum of the deeply-bound $K^{-} p p$ state.

\section{Acknowledgments}

We acknowledge Prof. M. Iwasaki, Dr. H. Ohta, Dr. H. Ohnishi and Dr. T. Suzuki for discussions about a plan of the J-PARC E15 experiment. We would like to thank Prof. Y. 
Akaishi for many valuable discussions and suggestions. This work is supported by Grantsin-Aid for Scientific Research on Priority Areas (Nos. 17070002, 17070007 and 20028012).

[1] D.B. Kaplan and A. E. Nelson, Phys, Lett. B175, 57 (1986);

G.E. Brown and M. Rho, Phys. Rep. 269, 333 (1996);

C.H. Lee, Phys. Rep. 275, 255 (1996).

[2] A. Doté, H. Horiuchi, Y.Akaishi, and T. Yamazaki, Phys. Rev. C70, 044313 (2004).

[3] Y. Nogami, Phys. Lett. 7, 288 (1963).

[4] T. Yamazaki and Y. Akaishi, Phys. Lett. B535, 70 (2002); Proc. Jpn. Academy, Series B 83, 144 (2007) (arXiv:0805.4382 [nucl-th]).

[5] N.V. Shevchenko, A. Gal, and J. Mareš, Phys. Rev. Lett. 98, 082301 (2007);

N.V. Shevchenko, A. Gal, J. Mareš, and J. Revai, Phys. Rev. C76, 044004 (2007).

[6] Y. Ikeda and T. Sato, Phys. Rev. C76, 035203 (2007).

[7] A. Doté, T. Hyodo, and W. Weise, Nucl. Phys. A804, 197 (2008); Phys. Rev. C79, 014003 (2009).

[8] A.N. Ivanov, P. Kienle, J. Marton, and E. Widmann, nucl-th/0512037.

[9] T. Nishikawa and Y. Kondo, Phys. Rev. C77, 055202 (2008).

[10] A. Arai, M. Oka, and S. Yasui, Prog. Theor. Phys. 119, 103 (2008).

[11] J. Yamagata, D. Jido, H. Nagahiro, and S. Hirenzaki, Mod. Phys. Lett. A23, 2528 (2008);

J. Yamagata-Sekihara, D. Jido, H. Nagahiro, and S. Hirenzaki, Phys. Rev. C80, 045204 (2009)

[12] S. Wycech and A.M. Green, Phys. Rev. C79, 014001 (2009).

[13] E. Oset and A. Ramos, Nucl. Phys. A635, 99 (1998); E. Oset, A. Ramos, and C. Bennhold, Phys. Lett. B527, 99 (2002).

[14] T. Hyodo and W. Weise, Phys. Rev. C77, 035204 (2008).

[15] M. Agnello et al., Phys. Rev. Lett. 94, 212303 (2005).

[16] V.K. Magas, E. Oset, A. Ramos, and H. Toki, Phys. Rev. C74, 025206 (2006).

[17] G. Bendiscioli et al., Nucl. Phys. A789, 222 (2007).

[18] T. Yamazaki et al., arXiv:0810.5182 [nucl-ex].

[19] M. Iwasaki et al., J-PARC proposal, http://j-parc.jp/NuclPart/pac_0606/pdf/p15-Iwasaki.pdf. 
[20] K. Suzuki et al., Proceedings of the International Conference on Exotic Atoms (EXA05), Austrian Academy of Science Press, Vienna, 83 (2005); K. Suzuki, talk at EXA08.

[21] T. Yamazaki and Y. Akaishi, Phys. Rev. C76, 045201 (2007).

[22] P. Kienle, Nucl. Phys. A804, 286 (2008).

[23] T. Koike and T. Harada, Phys. Lett. B652, 262 (2007).

[24] O. Morimatsu and K. Yazaki, Nucl. Phys. A483, 493 (1988); Prog. Part. Nucl. Phys. 33, 679 (1994).

[25] T. Harada, Phys. Rev. Lett. 81, 5287 (1998); Nucl. Phys. A672, 181 (2000); T. Harada and Y. Hirabayashi, Nucl. Phys. A759, 143 (2005).

[26] T. Kishimoto et al., Prog. Theor. Phys. 118, 181 (2007).

[27] J. Yamagata, H. Nagahiro, and S. Hirenzaki, Phys. Rev. C74, 014604 (2006); J. Yamagata and S. Hirenzaki, Eur. Phys. J. A31, 255 (2007).

[28] T. Koike and T. Harada, Nucl Phys. A804, 231 (2008).

[29] K. Ikuta, M. Arima, and K. Masutani, Prog. Theor. Phys. 108, 917 (2002).

[30] T. Koike and T. Harada, Mod. Phys. Lett. A23, 2540 (2008); ibid. A24, 911 (2009); Int. J. Mod. Phys. A24, 2126 (2009).

[31] J. Hüfner, S.Y. Lee, and H.A. Weidenmüller, Nucl. Phys. A234, 429 (1974).

[32] S. Tadokoro, H. Kobayashi, and Y. Akaishi, Phys. Rev. C51, 2656 (1995).

[33] T. Kishimoto, Phys. Rev. Lett. 83, 4701 (1999).

[34] G.P. Gopal et al., Nucl. Phys. B119, 362 (1977).

[35] A.S. Rosenthal and F. Tabakin, Rhys. Rev. C22, 711 (1980).

[36] C.B. Dover and A. Gal, Ann. Phys. (N.Y.) 146, 309 (1983).

[37] I. Angeli, Atomic Data and Nuclear Data Tables 87, 185 (2004).

[38] C. Amsler et al. (Particle Data Group), Phys. Lett. B667, 1 (2008).

[39] A. Cieplý, E. Friedman, A. Gal, and J. Mareš, Nucl. Phys. A696, 173 (2001).

[40] C.B. Dover, L. Ludeking, and G.E. Walker, Phys. Rev. C22, 2073 (1980).

[41] J. Mareš, E. Friedman and A. Gal, Phys. Lett. B606, 295 (2005); Nucl. Phys. A770, 84 (2006).

[42] D. Gazda, E. Friedman, A. Gal, and J. Mareš, Phys. Rev. C76, 055204 (2007); J. Mareš, Nucl. Phys. A804, 296 (2008).

[43] In Note added in proof of Ref. [11], it is commented that the bound states with $L \geq 1$ 
should not be interpreted as the three-body $K^{-} p p$ state, but could be $\Lambda(1405)$ formed by the two-body $K^{-} p$ state.

[44] Y. Ikeda and T. Sato, Phys. Rev. C79, 035201 (2009).

[45] H. Feshbach, Ann. of Phys. 5, 357 (1958); ibid. 19, 287(1962).

[46] Y. Akaishi, K.S. Myint, and T. Yamazaki, Proc. Jpn. Academy, Series B 84, 264 (2008) (arXiv:0805.4382 [nucl-th]).

[47] P.A. Katz, K. Bunnel, M. Derrik, T. Fields, L.G. Hyman, and G. Keys, Phys. Rev. D1, 1267 (1970).

[48] T. Onaga, H. Narumi and T. Kouhmura, Prog. Theor. Phys. 82, 222 (1989).

[49] T. Suzuki et al., Mod. Phys. Lett. A23, 2520 (2008); Phys. Rev. C76, 068202 (2007). 
TABLE I: Parameters of the real and imaginary strengths, $V_{0}$ and $W_{0}$, of the $K^{--}$" $p p$ " optical potentials $U^{\mathrm{opt}}(E ; \boldsymbol{r})$ for the $I=1 / 2, J^{\pi}=0^{-}$bound state in Eq.(17). The range parameter is $b=1.09 \mathrm{fm}$. The branching rates of the one-nucleon $K^{-}$absorption process are taken to be $B_{1}^{(\pi \Sigma N)}=0.7$ and $B_{1}^{(\pi \Lambda N)}=0.1$, respectively, and the branching rate of the two-nucleon $K^{-}$ absorption process is $B_{2}^{(Y N)}=0.2$. The values in the brackets denote for the imaginary parts of the energy-independent potentials $U_{0}^{\text {opt }}(\boldsymbol{r})$. The unit of all values is $\mathrm{MeV}$.

\begin{tabular}{|c|c|c|c|c|c|c|c|}
\hline \multirow[t]{2}{*}{ Potentials } & \multirow[t]{2}{*}{$V_{0}$} & \multirow[t]{2}{*}{$W_{0}$} & \multicolumn{2}{|c|}{ without $B_{2}^{(Y N)}$} & \multicolumn{2}{|c|}{ with $B_{2}^{(Y N)}$} & \multirow[t]{2}{*}{ Refs. } \\
\hline & & & B.E. ${ }^{a}$ & $\Gamma^{b}$ & $B . E .^{a}$ & $\Gamma^{c}$ & \\
\hline $\mathrm{A}$ & -237 & $-128(-120)$ & 21 & 70 & 15 & 92 & DHW [7] \\
\hline B & -292 & $-107(-86)$ & 48 & 61 & 45 & 82 & YA [4] \\
\hline $\mathrm{C}$ & -344 & $-203(-147)$ & 70 & 110 & 59 & 164 & SGM [5] \\
\hline $\mathrm{D}_{1}$ & -399 & $-372(-86)$ & 114 & 34 & 105 & 118 & DISTO [18] \\
\hline $\mathrm{D}_{2}$ & -404 & $-213(-47)$ & 118 & 19 & 115 & 67 & FINUDA [15] \\
\hline $\mathrm{D}_{3}$ & -458 & $-82(-13)$ & 162 & 5 & 161 & 24 & OBELIX [17] \\
\hline
\end{tabular}

${ }^{a}$ Binding energy of the $K^{-} p p$ bound state measured from the $K^{-}+p+p$ threshold.

${ }^{b}$ Width from the one-nucleon $K^{-}$absorption processes.

${ }^{c}$ Total width from the one- and two-nucleon $K^{-}$absorption processes. 


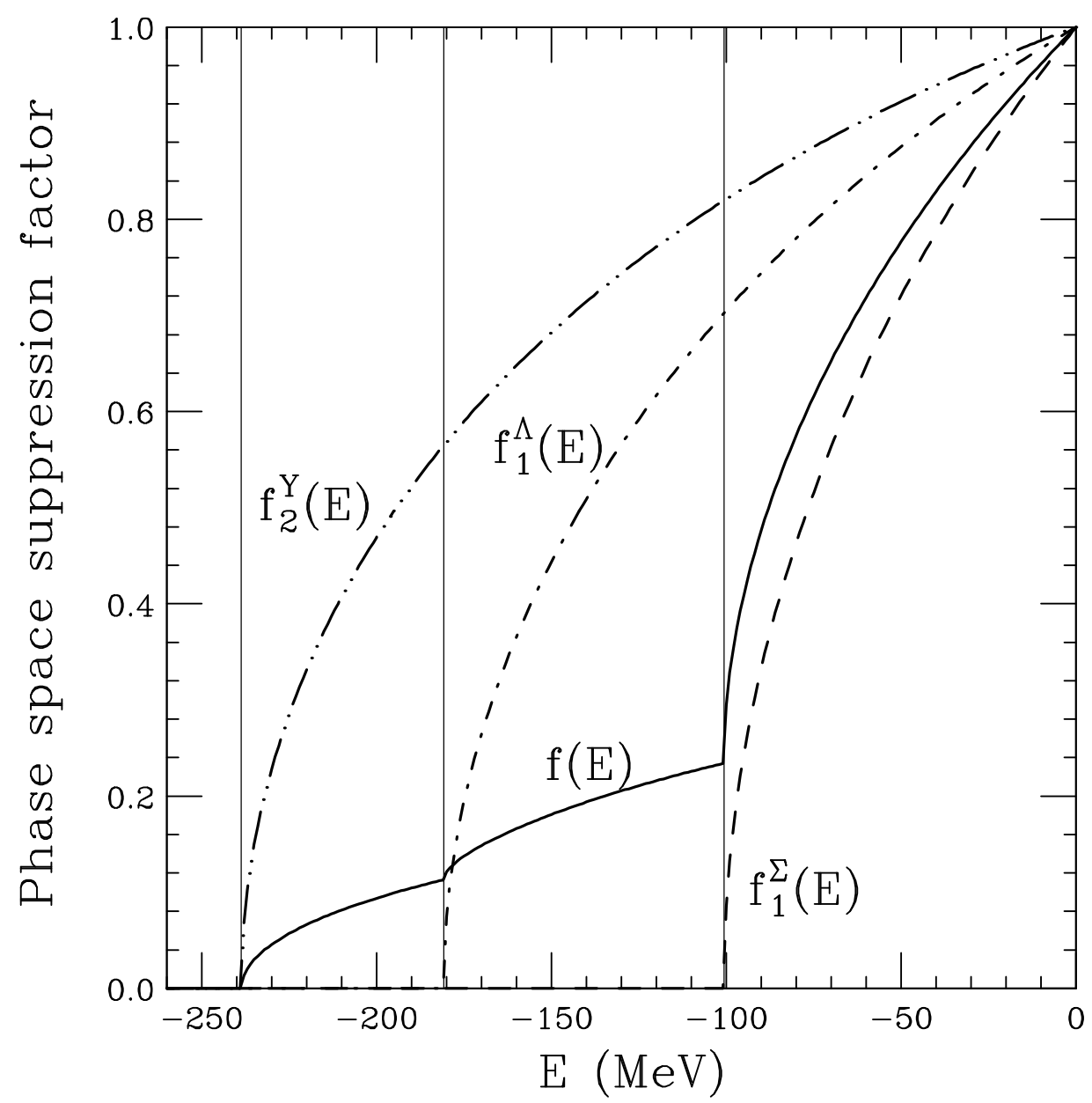

FIG. 1: Phase space suppression factors introduced by Mareš et al. [41], as a function of $E$. The dashed and dash-dotted curve denote the phase space factors $f_{1}^{\Sigma}(E)$ for the $\left[K^{-} p p\right] \rightarrow \pi+\Sigma+N$ process and $f_{1}^{\Lambda}(E)$ for the $\left[K^{-} p p\right] \rightarrow \pi+\Lambda+N$ one, respectively, from the one-nucleon $K^{-}$ absorption. The dash-dot-dotted curve denotes $f_{2}^{Y}(E)$ for the $\left[K^{-} p p\right] \rightarrow Y+N$ process from the two-nucleon $K^{-}$absorption. The solid curve denotes the total phase space factor $f(E)=$ $B_{1}^{(\pi \Sigma N)} f_{1}^{\Sigma}(E)+B_{1}^{(\pi \Lambda N)} f_{1}^{\Lambda}(E)+B_{2}^{(Y N)} f_{2}^{Y}(E)$, where $\left(B_{1}^{(\pi \Sigma N)}, B_{1}^{(\pi \Lambda N)}, B_{2}^{(Y N)}\right)=(0.7,0.1,0.2)$ is assumed. The vertical lines at $E \simeq-100 \mathrm{MeV},-180$ and $-240 \mathrm{MeV}$ indicate the $\pi+\Sigma+N$, $\pi+\Lambda+N$ and $Y+N$ decay threshold energies, respectively. 


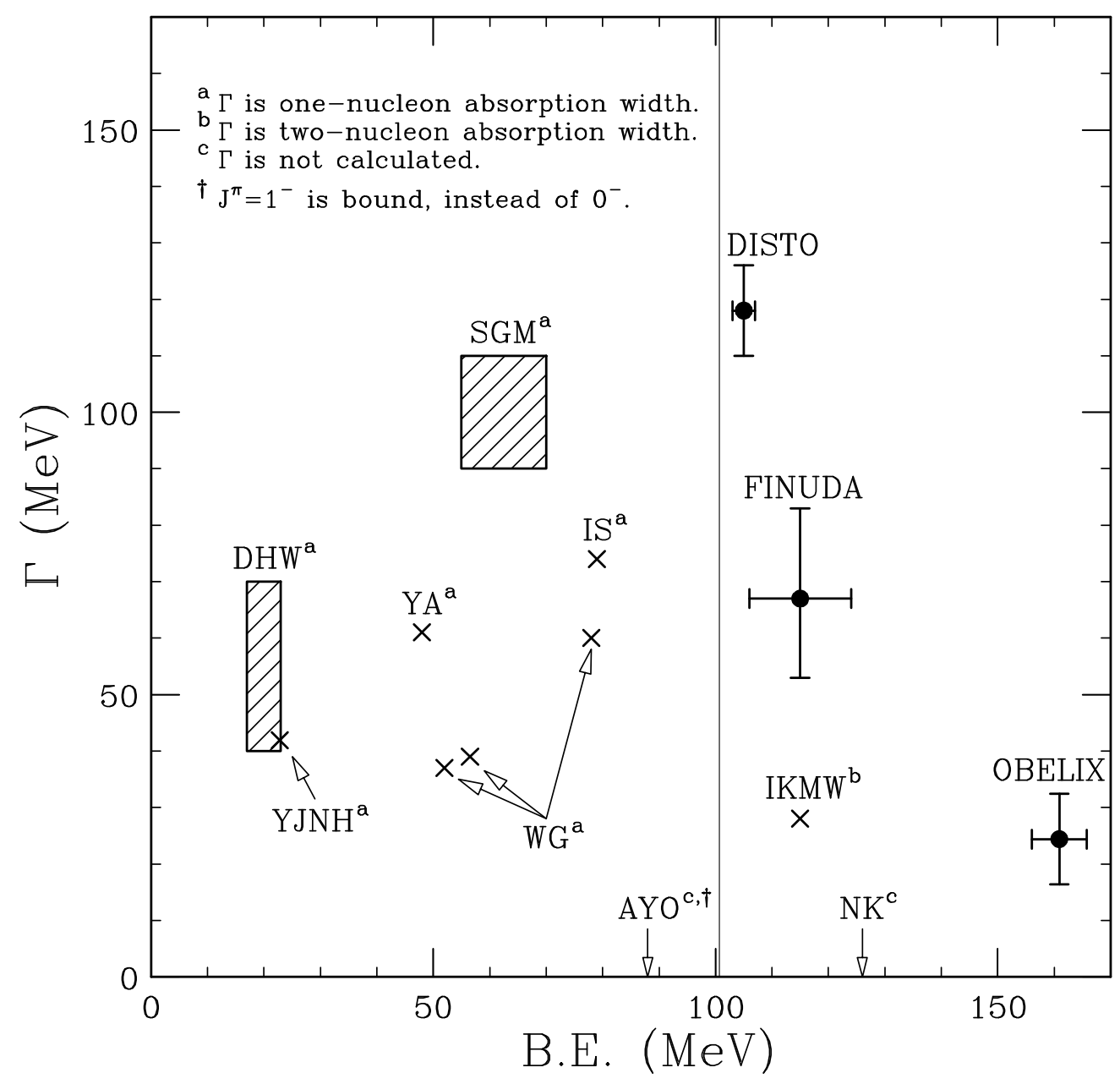

FIG. 2: Summary of the binding energies and widths of the $K^{-} p p$ bound state. Theoretical calculations with the $I=1 / 2, J^{\pi}=0^{-}$state are predicted by YA (Yamazaki, Akaishi) [4], SGM (Shevchenko, Gal, Mareš) [5], IS (Ikeda, Sato) [6], DHW (Doté, Hyodo, Weise) [7], IKMW (Ivanov, Kienle, Marton, Widmann) [8], NK (Nishikawa, Kondo) [9], YJNH (Yamagata, Jido, Nagahiro, Hirenzaki) [11], and WG (Wycech, Green) [12]; the $I=1 / 2, J^{\pi}=1^{-}$state by AYO (Arai, Yasui, Oka) [10]. The data are taken from the FINUDA [15], OBELIX [17] and DISTO [18] experiments. The vertical line at $B . E . \simeq 100 \mathrm{MeV}$ indicates the $\pi \Sigma N$ decay threshold. 


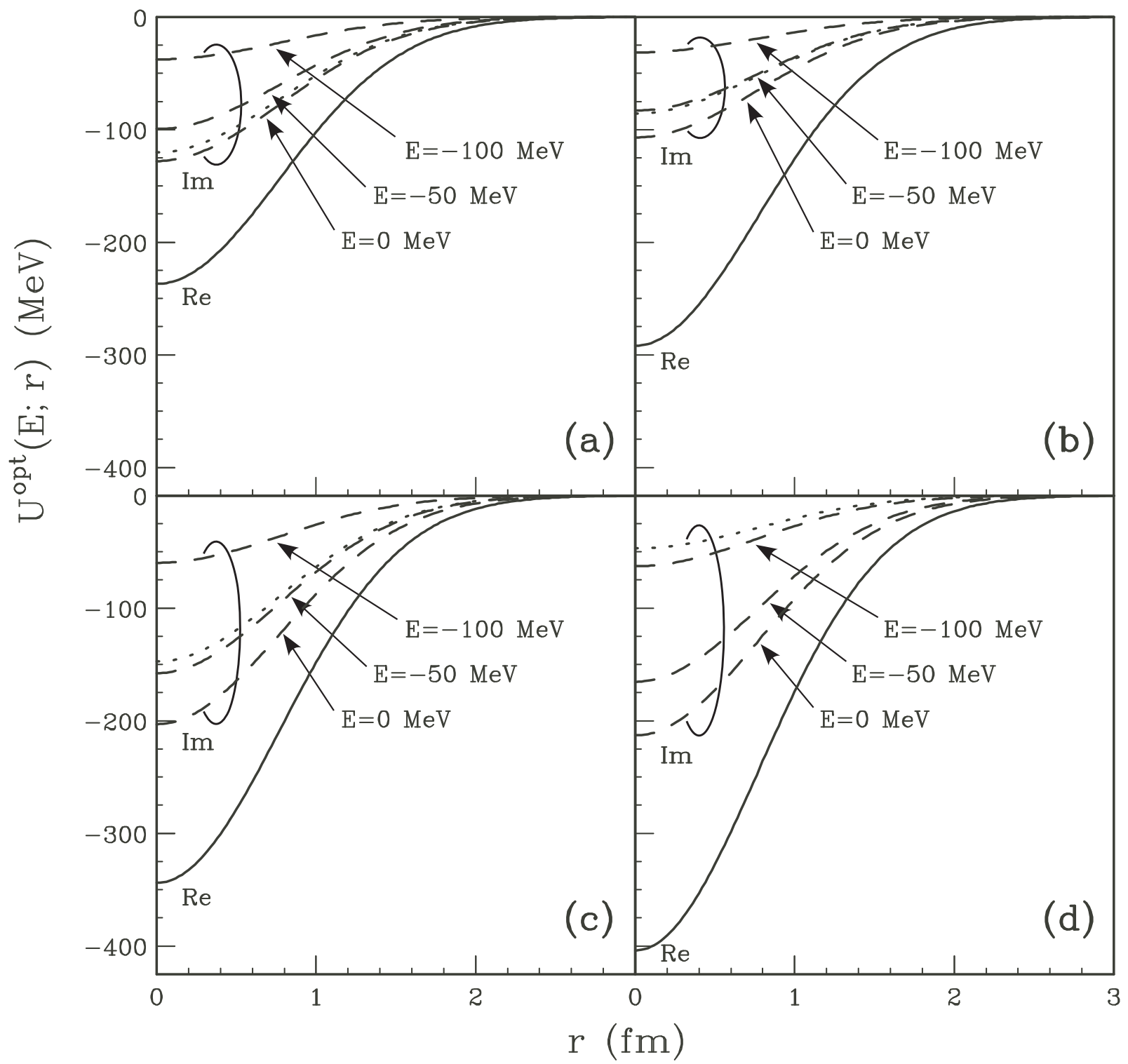

FIG. 3: Real and imaginary parts of the $K^{-}$- "pp" optical potentials $U^{\text {opt }}(E ; \boldsymbol{r})$ for potentials (a) A, (b) B, (c) C and (d) $\mathrm{D}_{2}$, as a function of a distance between the $K^{-}$and the center of the " $p p$ " core nucleus. The solid curves denote the real parts, and the dashed curves the imaginary parts at $E=0,-50$ and $-100 \mathrm{MeV}$. The dotted curves denote the imaginary parts for the energyindependent $K^{-}$- " $p p$ " optical potentials $U_{0}^{\mathrm{opt}}(\boldsymbol{r})$. 


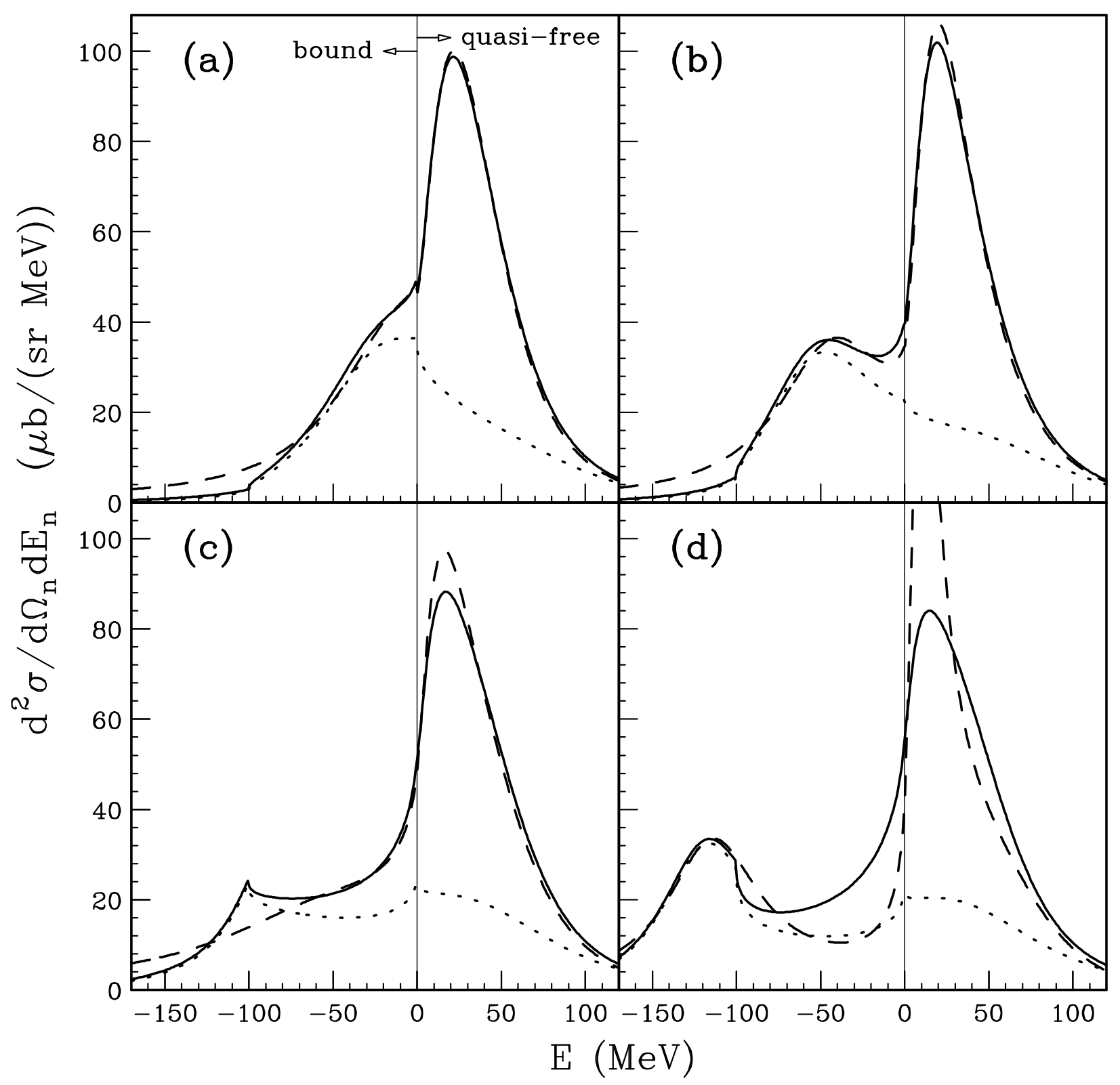

FIG. 4: Calculated inclusive spectra of the ${ }^{3} \mathrm{He}\left(\right.$ in-flight $\left.K^{-}, n\right)$ reaction at $p_{K^{-}}=1.0 \mathrm{GeV} / \mathrm{c}$ and $\theta_{\text {lab }}=0^{\circ}$ as a function of the energy $E$ of the $K^{-} p p$ system measured from $K^{-}+p+p$ threshold for potentials (a) A, (b) B, (c) C and (d) $\mathrm{D}_{2}$. The solid and dashed curves denote the inclusive spectra with the energy-dependent $U^{\mathrm{opt}}(E)$ and energy-independent $U_{0}^{\mathrm{opt}}$ potentials, respectively. The dotted curve denotes the $L=0$ component in the inclusive spectrum for $U^{\text {opt }}(E)$. The vertical line at $E=0 \mathrm{MeV}$ indicates the $K^{-}+p+p$ threshold, and the left- and right-hand sides of this line are the $K^{-}$bound and quasi-free scattering regions, respectively. 


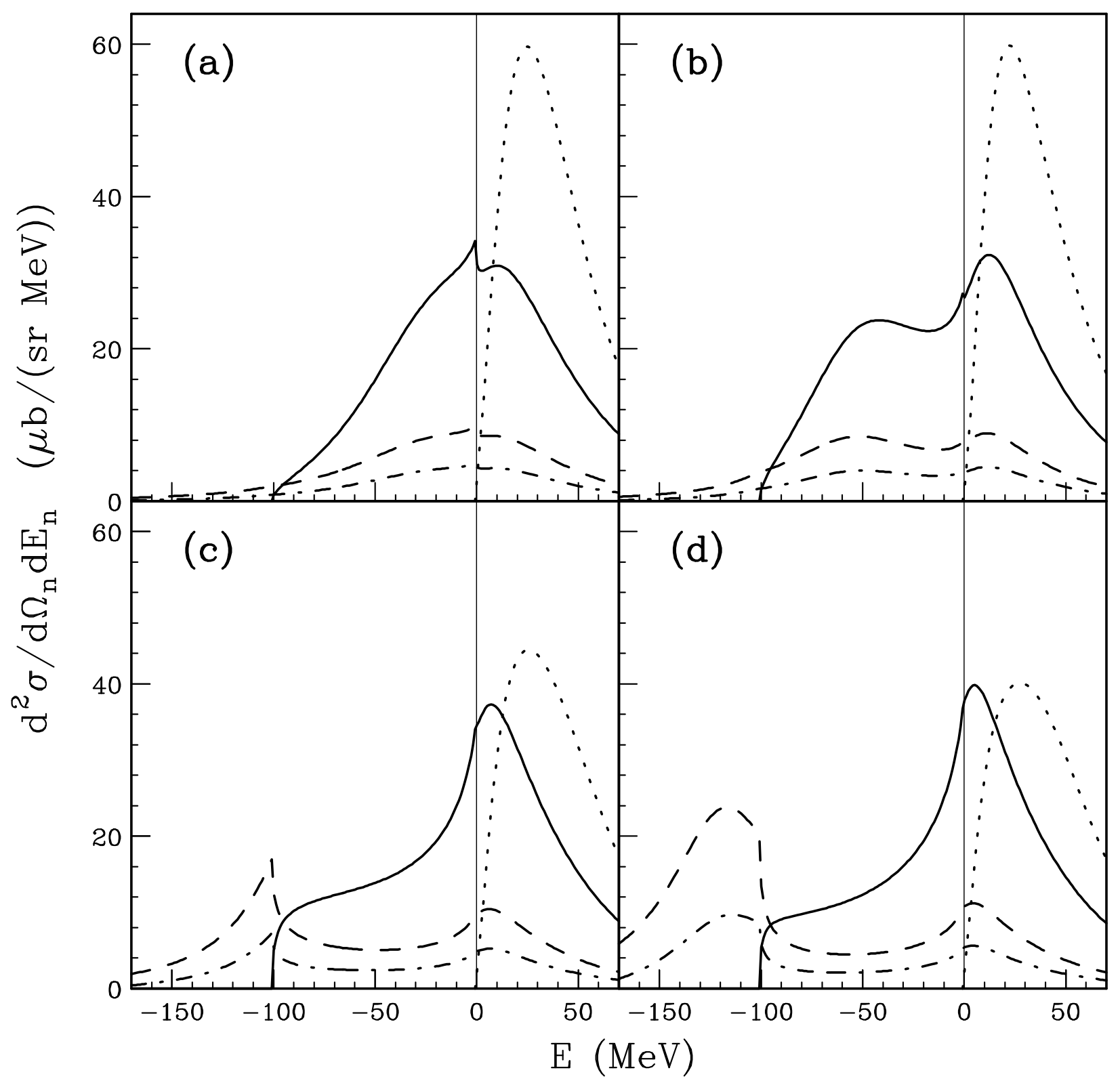

FIG. 5: Calculated semi-exclusive spectra of the ${ }^{3} \mathrm{He}\left(\right.$ in-flight $\left.K^{-}, n\right)$ reaction at $p_{K^{-}}=1.0 \mathrm{GeV} / \mathrm{c}$ and $\theta_{\text {lab }}=0^{\circ}$, for potentials (a) A, (b) B, (c) C and (d) $\mathrm{D}_{2}$. The solid and dot-dashed curves denote the $\left[K^{-} p p\right] \rightarrow \pi+\Sigma+N$ and $\pi+\Lambda+N$ decay processes from the one-nucleon $K^{-}$absorption, respectively. The dashed curves denote the $\left[K^{-} p p\right] \rightarrow Y+N$ decay process from the two-nucleon $K^{-}$absorption. The dotted curves denote the spectra of the $K^{-}$escape process. See also the caption in Fig. 4. 


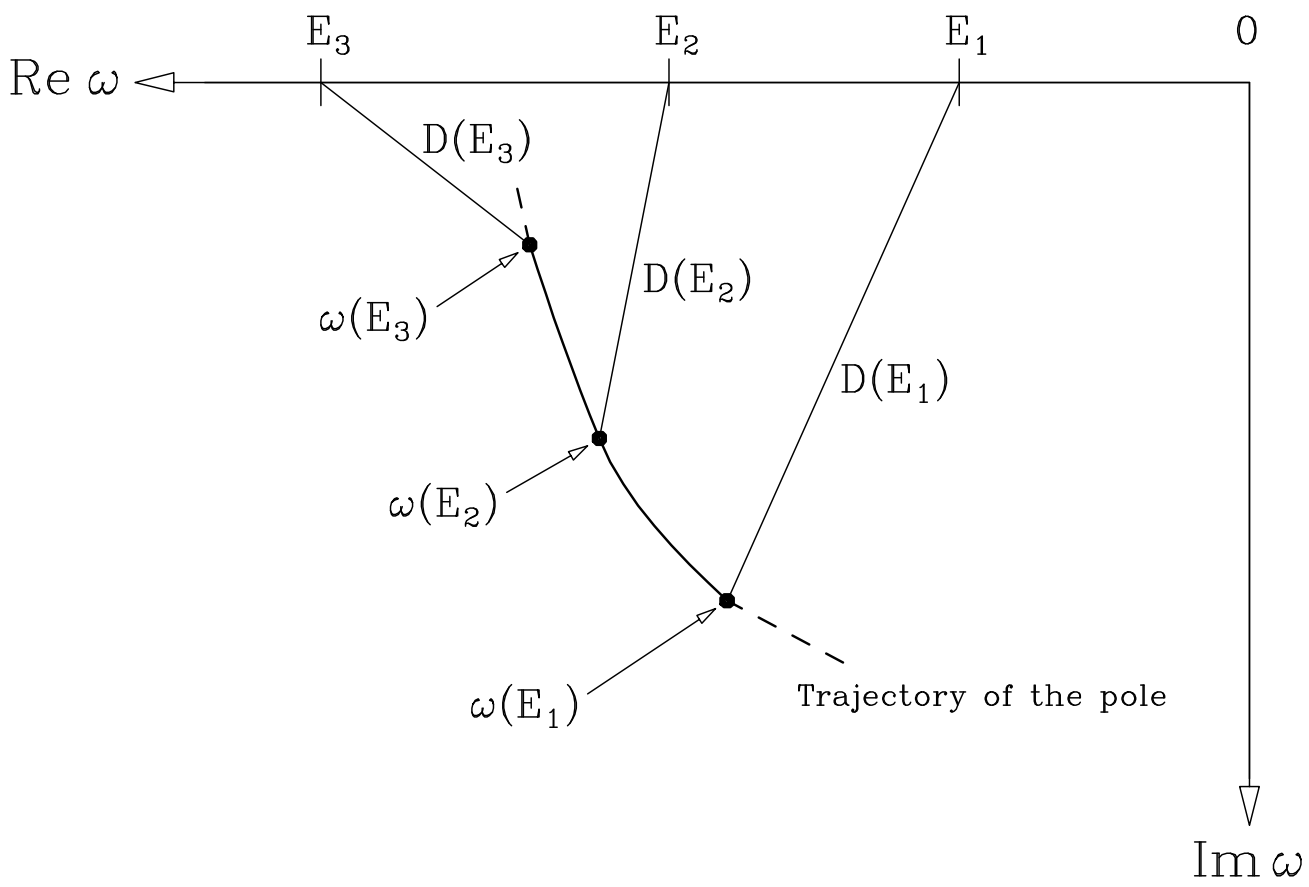

FIG. 6: Distance $D(E)$ between a point $(E, 0)$ on the real axis and a pole at $(\operatorname{Re} \omega(E), \operatorname{Im} \omega(E))$ for the $K^{-} p p$ state in the complex energy plane. The pole moves on the trajectory, as a function of the real energy $E$. Some examples are illustrated at $E=E_{i}(i=1,2,3)$. 

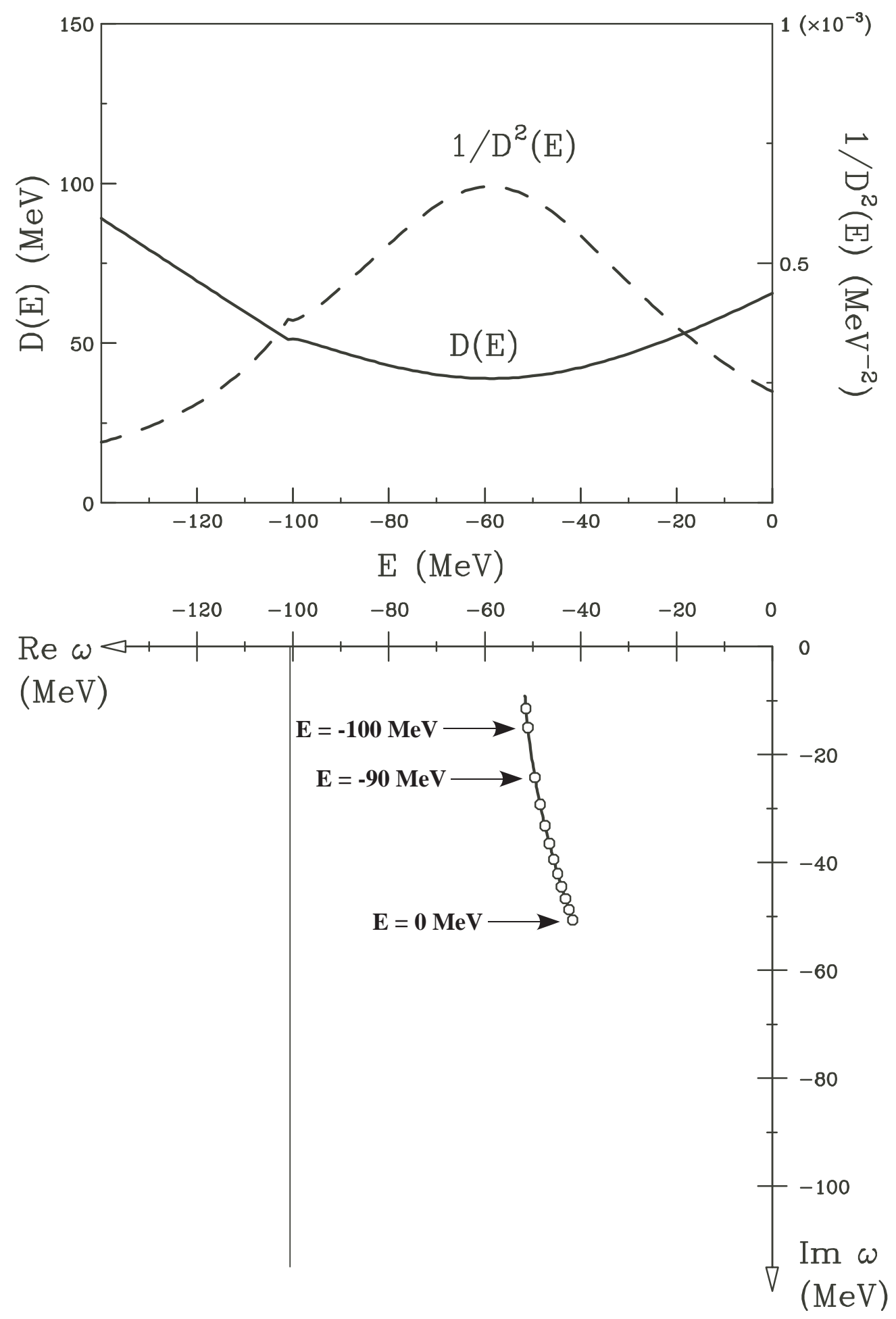

FIG. 7: Pole trajectory of the $K^{-} p p$ state (bottom) and $D(E)$ (top) in the complex energy plane, in the case of potential $\mathrm{B}\left(V_{0}=-292 \mathrm{MeV}\right.$ and $\left.W_{0}=-107 \mathrm{MeV}\right)$. The circles denote the pole positions at $\omega(E)$, which are drawn from $E=-110 \mathrm{MeV}$ to $0 \mathrm{MeV}$ in steps of $10 \mathrm{MeV}$. The dashed curve denotes $1 / D^{2}(E)$ which roughly represents the contribution of the pole in the spectrum. 

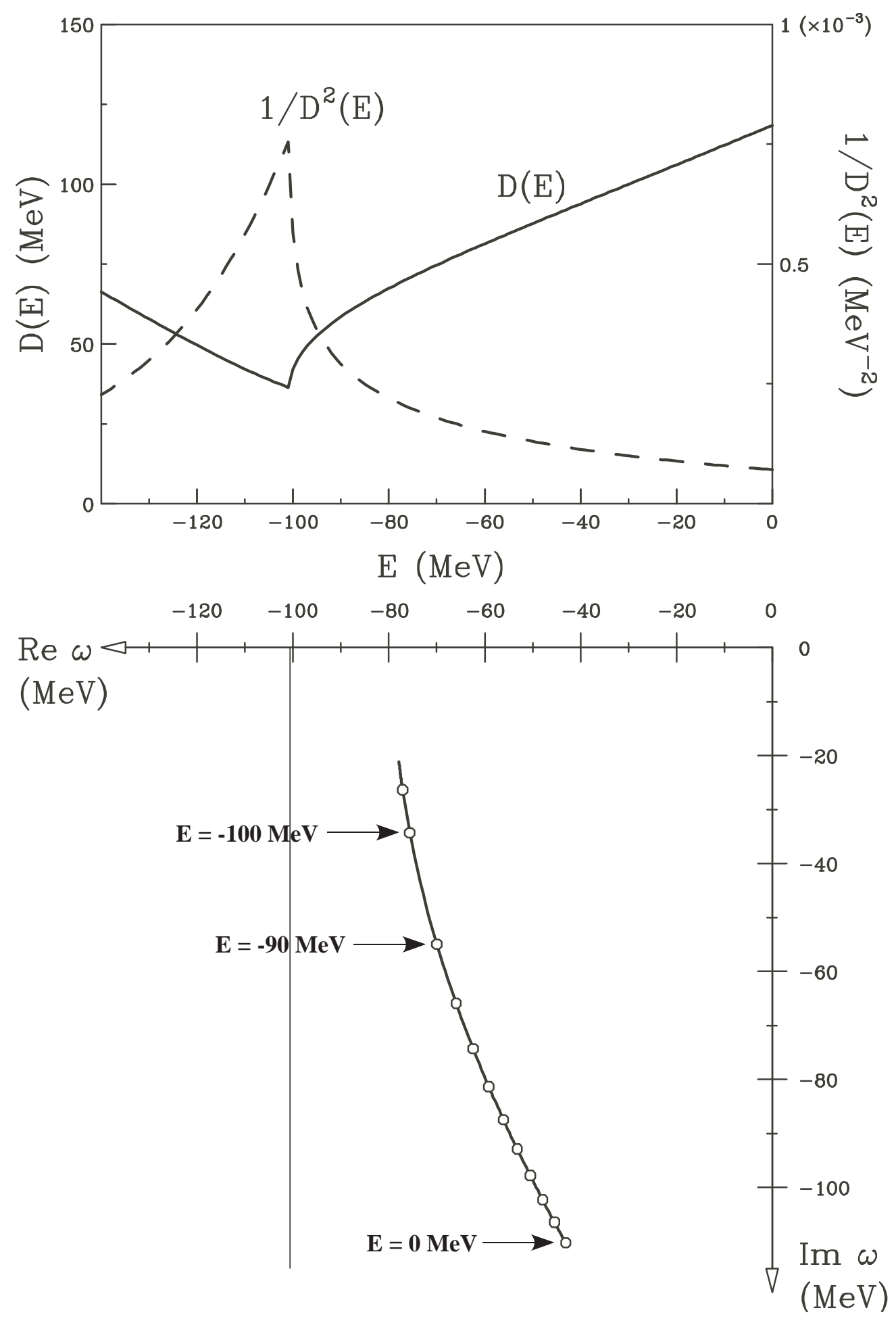

FIG. 8: Pole trajectory of the $K^{-} p p$ state and $D(E)$ in the complex energy plane, in the case of potential $\mathrm{C}\left(V_{0}=-344 \mathrm{MeV}\right.$ and $\left.W_{0}=-203 \mathrm{MeV}\right)$. See also the caption of Fig. 7 . 

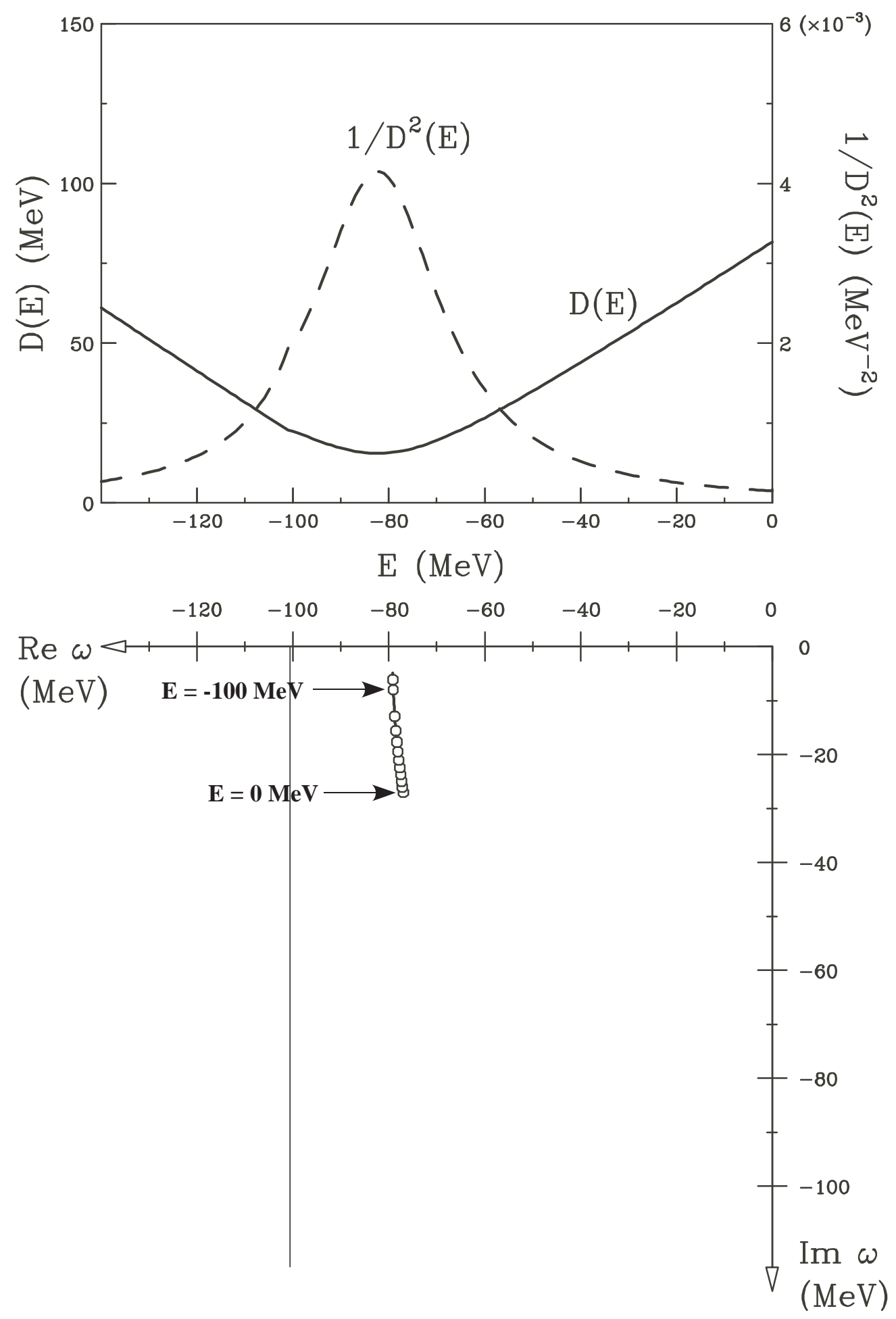

FIG. 9: Pole trajectory of the $K^{-} p p$ state and $D(E)$ in the complex energy plane, in the case of potential $\mathrm{C}$ modified with an artificial narrow width $\left(V_{0}=-344 \mathrm{MeV}\right.$ and $\left.W_{0}=-47 \mathrm{MeV}\right)$. See also the caption of Fig. 7. 


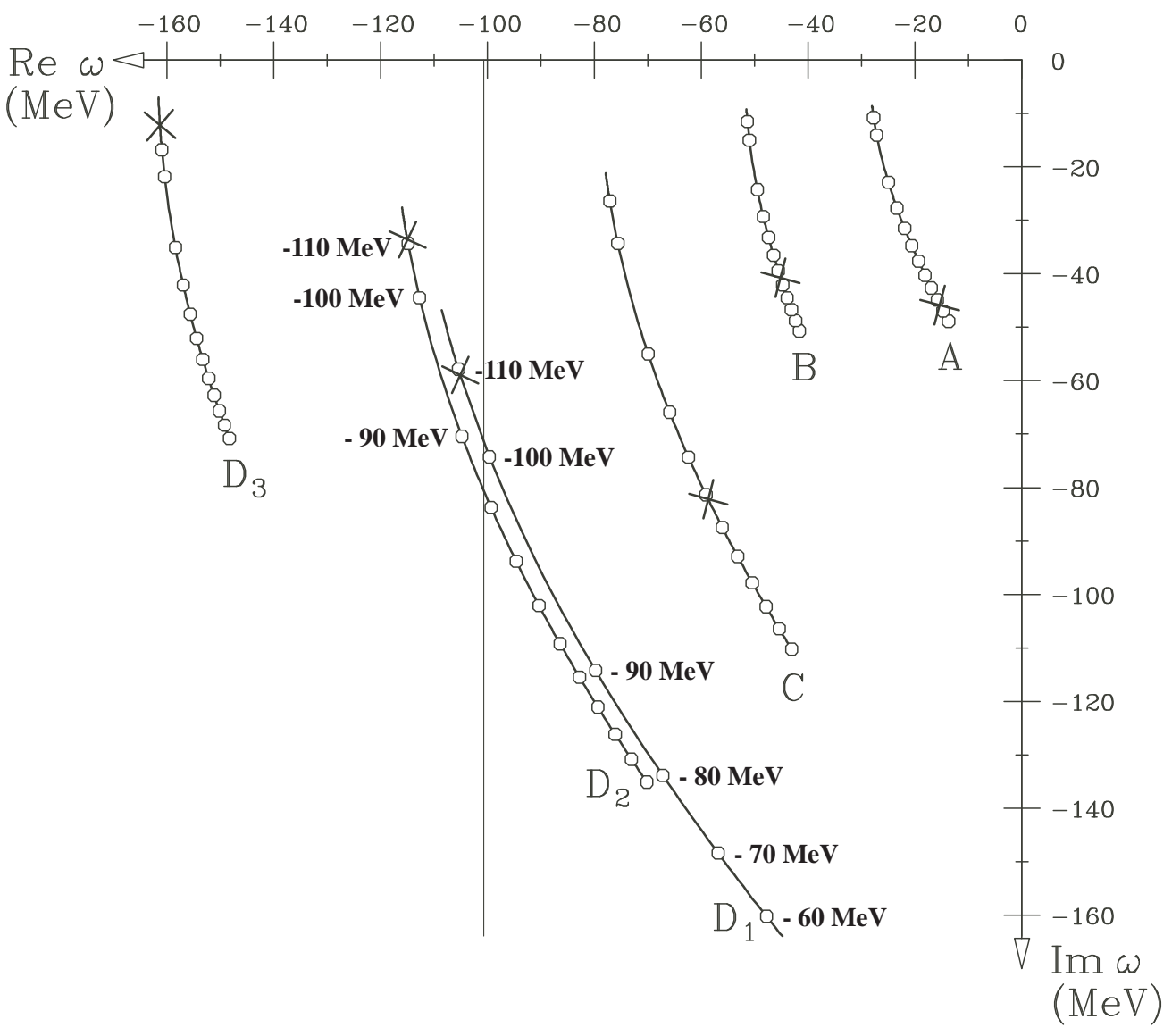

FIG. 10: Pole trajectories of the $K^{-} p p$ state for potentials $\mathrm{A}, \mathrm{B}, \mathrm{C}, \mathrm{D}_{1}, \mathrm{D}_{2}$ and $\mathrm{D}_{3}$ in the complex energy plane. The circles denote the pole positions at $\omega(E)$, which are drawn from $E=-110 \mathrm{MeV}$ to $0 \mathrm{MeV}$ in steps of $10 \mathrm{MeV}$. The crosses denote positions at $(-B . E .,-\Gamma / 2)$ of which values are given in Table I with these potentials. 


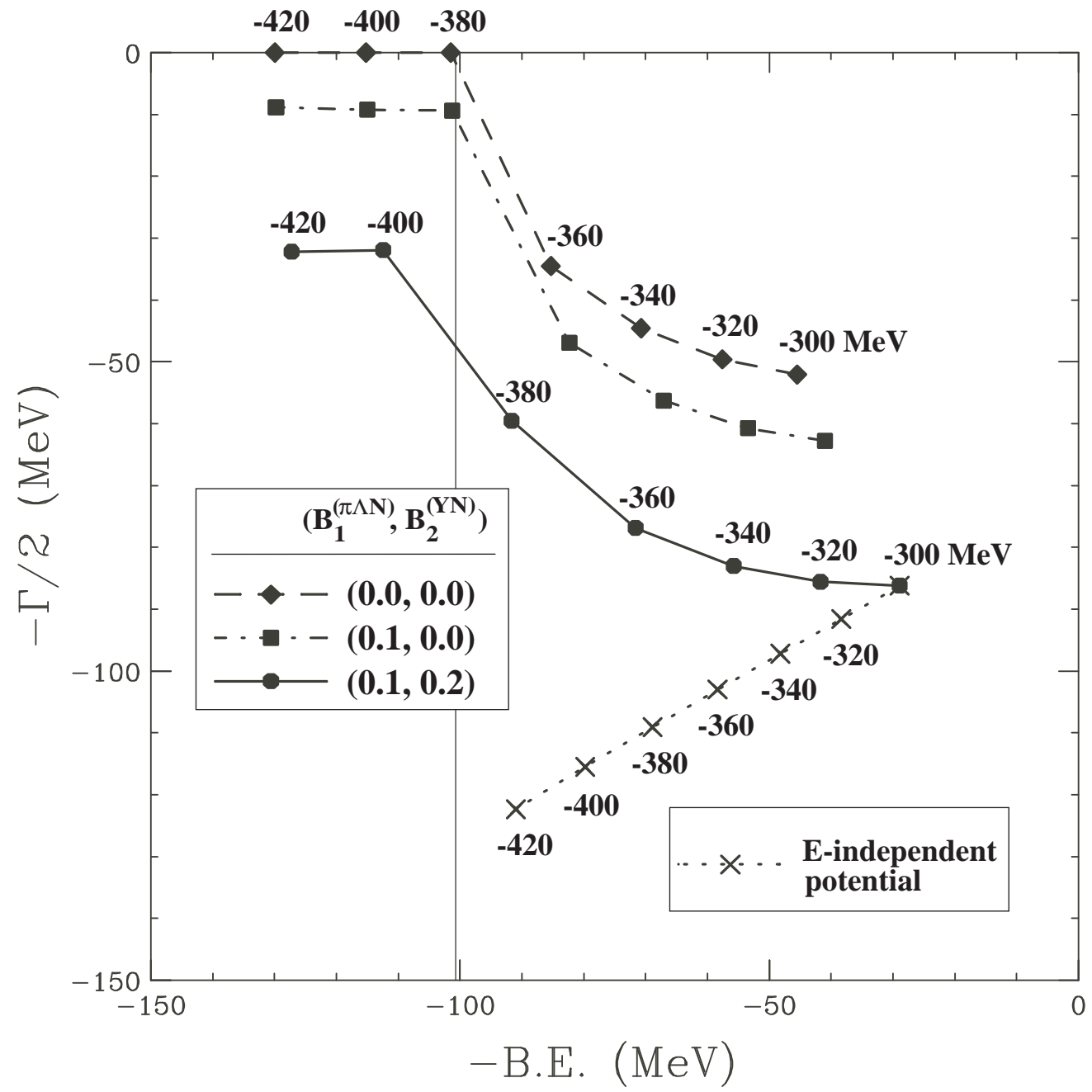

FIG. 11: The behavior of the moving pole at $(-B . E .,-\Gamma / 2)$ when the value of $V_{0}$ for the energydependent potential $U^{\mathrm{opt}}(E)$ is changed. The diamonds, squares and circles denote the cases of $\left(B_{1}^{(\pi \Lambda N)}, B_{2}^{(Y N)}\right)=(0.0,0.0),(0.1,0.0)$ and $(0.1,0.2)$ in $U^{\text {opt }}(E)$ with $B_{1}^{(\pi \Sigma N)}=0.7$ and $W_{0}=$ $-203 \mathrm{MeV}$, respectively. The crosses denote the case of the energy-independent potential $U_{0}^{\text {opt }}$ with $W_{0}=-179 \mathrm{MeV}$. The numbers attached to the symbols give the corresponding values of $V_{0}$. 


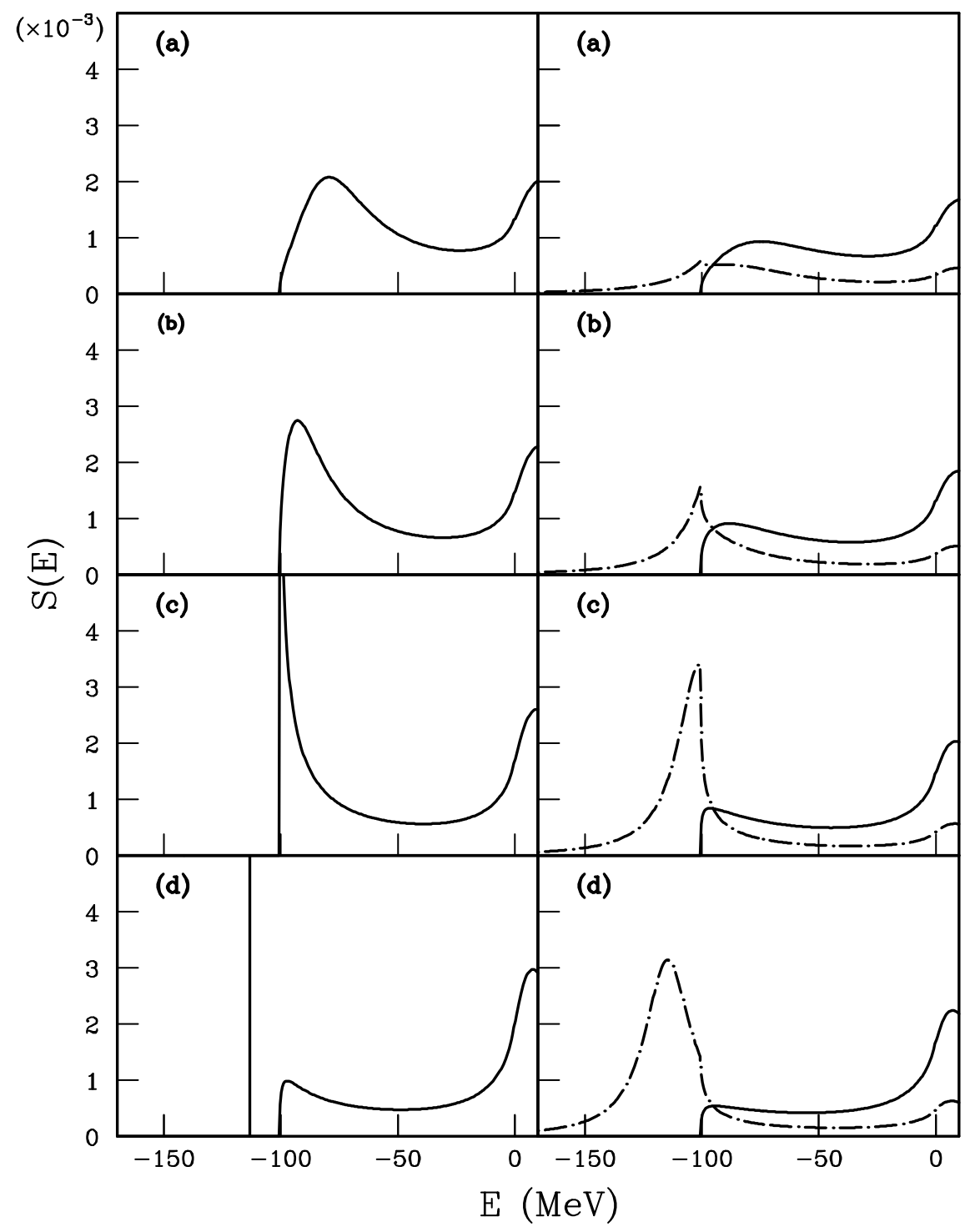

FIG. 12: Behavior of the strength function $S(E)$ at $W_{0}=-107 \mathrm{MeV}$ when changing the value of $V_{0}$; (a) $V_{0}=-340 \mathrm{MeV}$, (b) $-360 \mathrm{MeV}$, (c) $-380 \mathrm{MeV}$ and (d) $-400 \mathrm{MeV}$ in (left) the case of $\left(B_{1}^{(\pi \Sigma N)}, B_{2}^{(Y N)}\right)=(0.7,0.0)$ and (right) the case of $\left(B_{1}^{(\pi \Sigma N)}, B_{2}^{(Y N)}\right)=(0.7,0.2)$. The solid and dashed curves denote $S_{\pi \Sigma N}^{\text {con }}(E)$ and $S_{Y N}^{\text {con }}(E)$, respectively. 


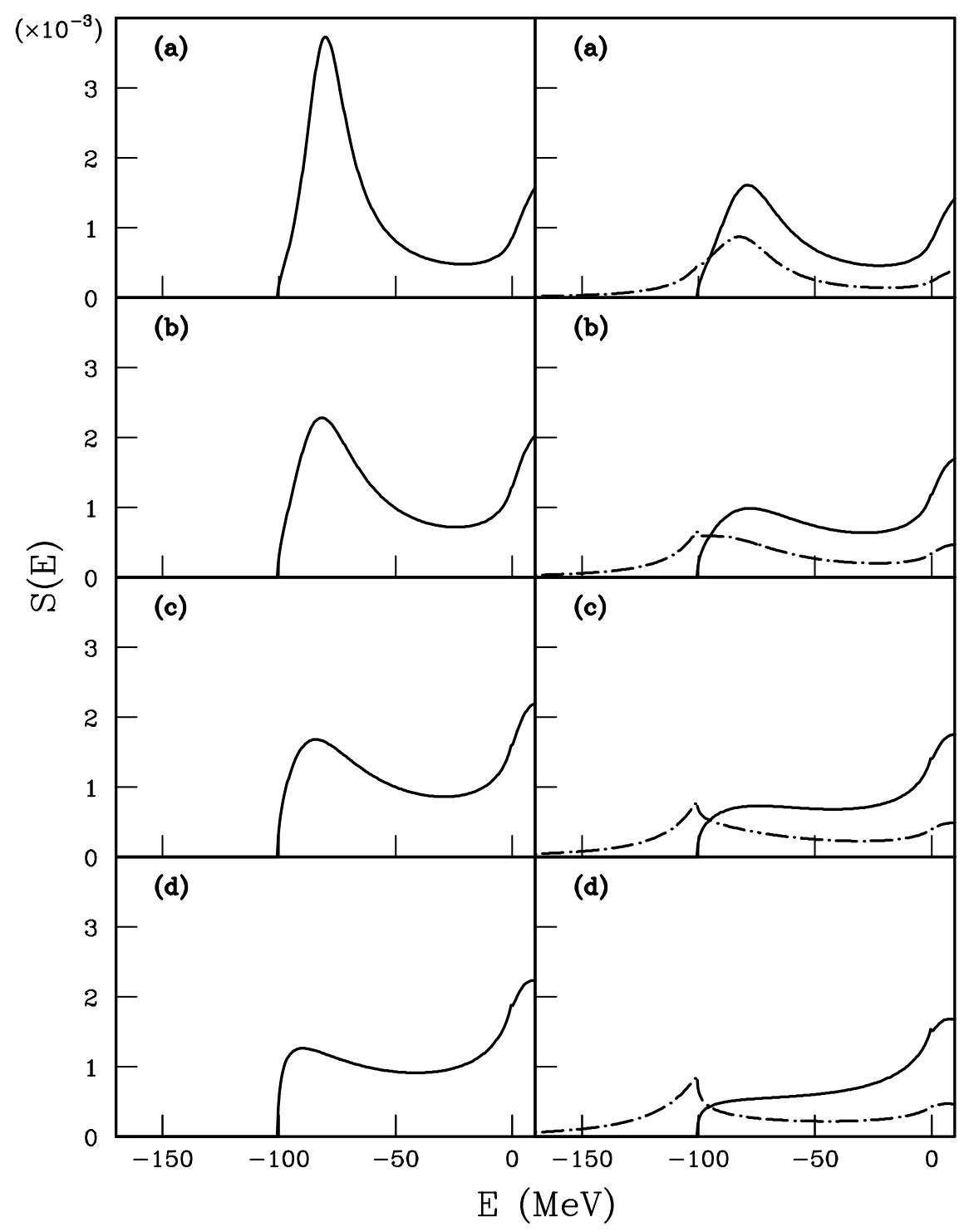

FIG. 13: Behavior of the strength function $S(E)$ at $V_{0}=-344 \mathrm{MeV}$ when changing the value of $W_{0}$; (a) $W_{0}=-60 \mathrm{MeV}$, (b) $-100 \mathrm{MeV}$, (c) $-140 \mathrm{MeV}$ and (d) $-200 \mathrm{MeV}$. See also the caption in Fig. 12. 


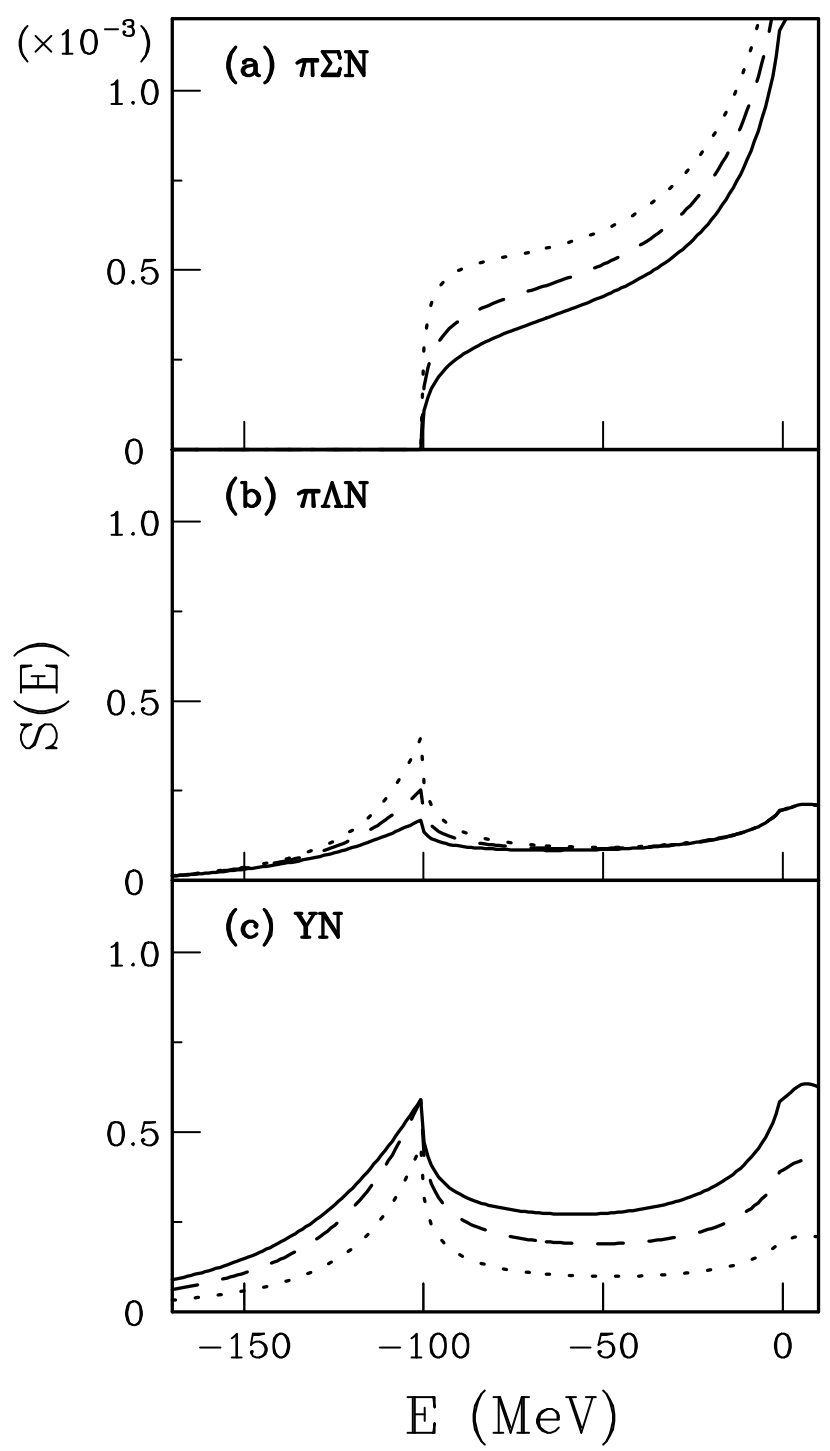

FIG. 14: Behavior of the calculated strength functions $S(E)$ for (a) $\left[K^{-} p p\right] \rightarrow \pi+\Sigma+N$, (b) $\left[K^{-} p p\right] \rightarrow \pi+\Lambda+N$ and $(\mathrm{c})\left[K^{-} p p\right] \rightarrow Y+N$ decay processes. Here potential $\mathrm{C}$ with $B_{1}^{(\pi \Lambda N)}=0.1$ is used. The dotted, dashed and solid curves denote the spectra for $\left(B_{1}^{(\pi \Sigma N)}, B_{2}^{(Y N)}\right)=(0.8,0.1)$, $(0.7,0.2)$ and $(0.6,0.3)$, respectively. 